\title{
PERIOD phosphoclusters control temperature compensation of the Drosophila circadian clock
}

Radhika Joshi*.1, Yao D. Cai*,2, Yongliang Xia ${ }^{1}$, Joanna C. Chiu² and Patrick Emery ${ }^{1, \#}$

1 Department of Neurobiology, University of Massachusetts Chan Medical School, 364

Plantation street, Worcester, MA 01605

2 Department of Entomology and Nematology, University of California Davis, One Shields Avenue, Davis, CA 95616

* Equal contribution

\#Corresponding author: Patrick.Emery@umassmed.edu Phone: 508-856-6599 


\section{Abstract}

Temperature compensation is a critical feature of circadian rhythms, but how it is achieved remains elusive. Here, we uncovered the important role played by the Drosophila PERIOD (PER) phosphodegron in temperature compensation. Using CRISPR-Cas9, we introduced a series of mutations that altered three Serines (S44, 45 and 47 ) belonging to the PER phosphodegron, the functional homolog of mammalian PER2's S487 phosphodegron, which impacts temperature compensation. While all three Serine to Alanine substitutions lengthened period at all temperatures tested, temperature compensation was differentially affected. S44A and S45A substitutions caused decreased temperature compensation, while S47A resulted in overcompensation. These results thus reveal unexpected functional heterogeneity of phosphodegron residues in thermal compensation. Furthermore, mutations impairing phosphorylation of the pers phosphocluster decreased thermal compensation, consistent with its inhibitory role on S47 phosphorylation. Interestingly, the S47A substitution caused increased accumulation of hyper-phosphorylated PER at warmer temperatures. This finding was corroborated by cell culture assays in which S47A resulted in excessive temperature compensation of phosphorylation-dependent PER degradation. Thus, we show a novel role of the PER phosphodegron in temperature compensation through temperaturedependent modulation of the abundance of hyper-phosphorylated PER. Our work also reveals interesting mechanistic convergences and differences between mammalian and Drosophila temperature compensation of the circadian clock.

\section{Author summary:}

Circadian rhythms are critical adaptive mechanisms that enable most organisms to adjust their physiology and behavior to the changes that occur in their environment every day. Ambient temperature varies constantly, but interestingly molecular circadian pacemakers do not accelerate with increasing temperature, while most biochemical reactions are sensitive to temperature. This phenomenon of circadian temperature compensation is poorly understood. Using genome editing and transgenic approaches, we found that two phosphorylated motifs in the Drosophila PERIOD protein, which regulate stability, impact 
temperature compensation. Moreover, we observed that mutation of a key Serine residue controlling PER degradation, S47, affects the accumulation of phosphorylated PER in a temperature-dependent manner, and causes PER degradation kinetics to become overly protected from increased temperature. As a result, the circadian clock of S47 mutant flies is excessively temperature-compensated. Our work thus reveals an interesting mechanism that controls temperature compensation in Drosophila. Moreover, comparison with mammals reveal interesting similarities, but also important differences in how temperature compensation of the circadian clock is achieved. 


\section{Introduction:}

Circadian clocks are present across almost all life forms and allow them to adapt their activities with daily changes in their surrounding environment. In eukaryotes, the molecular circadian pacemaker is a self-sustained transcriptional feedback loop that repeats itself every $\sim 24 \mathrm{~h}$, thus producing molecular, physiological and behavioral rhythms that closely match day length (1). In Drosophila, the circadian clock comprises four core components (2). The transcription factors CLOCK (CLK) and CYCLE (CYC) form a heterodimer that binds to E-box motifs upstream of the period (per) and timeless (tim) genes to promote their transcription. PER and TIM heterodimerize in the cytoplasm before entering the nucleus. Once inside the nucleus, the PER/TIM heterodimer binds to CLK/CYC and thus inhibits per and tim transcription. PER, TIM, and CLK undergo various post-translational modifications that affect the period of the clock, the most notable being the progressive phosphorylation driven by kinases such as DOUBLETIME (DBT), SHAGGY, CASEIN KINASE 1 alpha (CK1 $\alpha$ ) (3), CASEIN KINASE 2 (CK2) and NEMO (NMO) (4).

Circadian clocks exhibit three key properties (5). First, these clocks free-run with a period of $\sim 24 \mathrm{~h}$ in the absence of external temporal cues (referred to as Zeitgebers) such as the daily light and temperature cycles. Second, these Zeitgebers can reset the clocks, thus anchoring the clock's phase to the day/night cycle. Third, the period of circadian clocks is temperaturecompensated. As ambient temperature increases, most enzymatic reactions speed up. However, under constant conditions, circadian clocks exhibit $\sim 24 \mathrm{~h}$ periodicities over a wide range of physiological temperatures, even in ectotherms. This was first observed by $C$. Pittendrigh while studying eclosion rhythms of Drosophila pseudoobscura (6).

The mechanism of temperature compensation is one of the long lasting mysteries of circadian rhythms. Different models have been proposed. For example, the network model proposes that multiple temperature-sensitive reactions cancel each other with changes in temperature. Early studies on Dinoflagellates indeed suggested that two temperature-sensitive reactions with opposite effects would help achieving constant period (7). At the biochemical level, phosphorylation mediated by CK1 and CK2 has received the most attention across species. Mutations in the $\beta 1$ and $\alpha$ subunits of CK2 in Neurospora alter temperature compensation (8). Temperature compensation in Arabidopsis is modulated by the inhibitory effect of CK2 
phosphorylation on CIRCADIAN ASSOCIATED CLOCK A1 (CCA1) binding. CCA1 binding to its target promoters increases with temperature, which is kept in check by the opposing effect

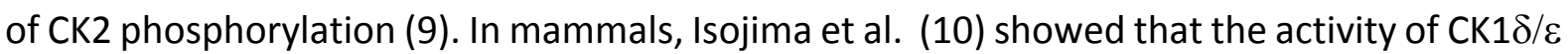
enzyme on synthetic PER2 peptide is temperature-insensitive, thus providing buffering

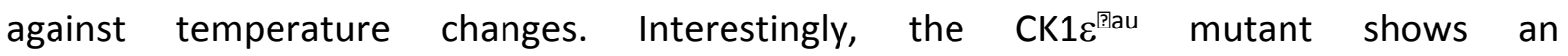
undercompensation phenotype $(11,12)$. Furthermore, CK1 $\delta / \varepsilon$ activity on PER2 results in a temperature-dependent phosphoswitch involving two phosphorylation sites (12-14). One of them is Serine (S)-478, also called phosphodegron. $S 478$ phosphorylation causes $\beta \operatorname{TrCP}$ (betatransducing repeat containing homolog protein)-dependent ubiquitination and degradation of mPER2 (13-15). The second is S659, the mouse homolog to the site of familial advanced sleep phase syndrome (FASPS) mutations (16). $S 659$ phosphorylation inhibits phosphorylation of the phosphodegron, and thus PER2 degradation $(12,17)$. S478 is preferentially phosphorylated at colder temperatures while FASPS domain phosphorylation increases at warmer temperatures, consequently balancing out PER stability $(12,18)$. Along with phosphorylation, other processes such as sumoylation in plants (19), nuclear cytoplasmic ratio of PER (20) and TIM (21), PER intermolecular and PER-TIM interactions (22-24) have all been proposed to modulate temperature compensation of circadian rhythms.

Over the past several decades circadian mutants have fuelled our understanding of the molecular clockwork. Therefore, to gain better understanding into the mechanism of temperature compensation in Drosophila we turned towards Drosophila mutants known to have a temperature compensation defect. Interestingly, of the numerous mutations impacting circadian rhythm period, only a subset of mutants show a defect in temperature compensation (25-32). We were intrigued by the mutant per ${ }^{\mathrm{SLIH}}$ (Some Like It Hot, Figure 1A) due to its striking undercompensation phenotype of $\sim 1.5 \mathrm{~h}$ when temperature is increased form $18^{\circ} \mathrm{C}$ to $29^{\circ} \mathrm{C}(31)$. The per $r^{S L H}$ mutation causes a Serine (S) to Tyrosine (Y) substitution at the $45^{\text {th }}$ residue, which is a part of Drosophila PER's phosphodegron (S44, 45 and 47) (33). The PER phosphodegron is regulated in a similar manner as its mammalian homolog (34)): it is phosphorylated by the CK1 $\delta / \varepsilon$ homolog DBT $(33,35,36)$, and its phosphorylation is inhibited by the pershort $\left(\mathrm{per}^{\varsigma}\right)$ phosphocluster, which is equivalent to the mammalian FASPS domain (12). Phosphorylation events at the PER phosphodegron, S47 in particular, are critical for 
SLIMB-mediated PER degradation and thus period length $(33,37,38)$. SLIMB is the fly homolog of $\beta \operatorname{TrCP}$. Here, we show that, unexpectedly, the residues of the Drosophila phosphodegron are functionally heterogeneous and differentially modulate temperature compensation, even though they all lengthen circadian period. In addition, we found that the per ${ }^{s}$ phosphocluster, which negatively regulates $\$ 47$ phosphorylation, also regulates temperature compensation. Furthermore, we show that substituting PER's S47 residue to an Alanine (A) results in an overcompensated circadian period that correlate with increased hyper-phosphorylated PER levels in vivo at warm temperature and overcompensated PER degradation in a wellestablished cell culture model. We therefore propose that PER phosphorylation and subsequent degradation is critical to temperature compensation in Drosophila. 


\section{Results:}

\section{Differential impact of three PER phosphodegron residues on temperature compensation}

The unique location of the per ${ }^{S L I H}$ mutation (Figure $1 \mathrm{~A}$ ) prompted us to evaluate the role of residues of the PER phosphodegron in temperature compensation. DBT, a Serine/Threonine kinase, phosphorylates S47, with S44 and S45 being additional potential targets (33). Thus, it would seem likely that phosphorylation at these residues modulate temperature compensation. To explore this possibility, we made phosphomimics ( $S$ to $D$ (Aspartate) or $E$ (Glutamate)) and phosphoinhibitors (S to Alanine (A)) at the PER S44, S45, and S47 using CRISPR-CAS9 (Figure 1A). Moreover, we generated S45F (S to Phenylalanine) to mimic contribution of a bulky hydrophobic amino acid similar to Tyrosine $(\mathrm{Y})$ in $\mathrm{per}^{\mathrm{SLIH}}$. We used S45S as a control, which contains only the silent substitutions introduced at the protospacer adjacent motifs (PAM site) during CRISPR mutagenesis. In our hands $w^{1118}$ and S45S control strains showed a decrease in period of $\sim 0.9 \mathrm{~h}$ (Table 1) over the range of 18 to $29^{\circ} \mathrm{C}$. To accurately represent the effect of mutations on temperature compensation, values plotted on figure $1 \mathrm{~B}$ are relative to the average of the two S45S control strains, which was set to 0 .

Consistent with previous observations (33), we observed that the phosphoinhibitory substitution S47A produced lengthened period. In addition, we found that S44A, S45A, S45F also resulted in longer periods, albeit to different extent at $25^{\circ} \mathrm{C}$ (Figure $1 \mathrm{~B}$, Table 1). S44A lengthens the period by $\sim 1.5 \mathrm{~h}, \mathrm{~S} 45 \mathrm{~A}$ by $2.5 \mathrm{~h}$, and $\mathrm{S} 47 \mathrm{~A}$ by $6.5 \mathrm{~h}$ at $25^{\circ} \mathrm{C}$. Interestingly, the effect on temperature compensation did not correlate with period length. S44A and S45A/F were undercompensated by $\sim 1$ to $2 \mathrm{~h}$, compared to controls. However, S47A showed an opposite phenotype: it caused a strong overcompensation of more than $2 \mathrm{~h}$ (Figure 1B).

Since it is possible that mutations at a residue affect phosphorylation events at the neighboring residues, we generated the double mutants S44-45A and S45-47A. S44-45A double mutants exhibited a very long period $(>34 \mathrm{~h})$ at $25^{\circ} \mathrm{C}$. Period was very long, but the effect on temperature compensation was difficult to interpret due to poor rhythmicity, particularly at $18^{\circ} \mathrm{C}$ (Table 1). However, the synergistic effects on period and rhythmicity indicate that S44 and 45 work together to control circadian rhythms. S45-47A mutants were completely arrhythmic at both temperatures tested (Table 1). 
We also tested phosphomimic substitutions (S to D/E). S45D and S45E did not show any substantial effect on either period length or temperature compensation (Figure 1, Table 1). S44D lengthened the period to $26.6 \mathrm{~h}$ at $25^{\circ} \mathrm{C}$, but had no effect on temperature compensation The behavior of S47D was the most peculiar. It showed a short rhythm period phenotype (>21h), as previously reported by Chiu et al (33) with per genomic rescue constructs in a per ${ }^{0}$ background. However, period was overcompensated by more than $2 \mathrm{~h}$. This could either be because phosphomimics do not correctly reproduce the structure of a phosphorylated residue (only its charge), or because phosphomimics are neither temporally controlled nor reversible and could thus have unexpected impact on temperature compensation.

Thus, taken together our results show that the PER phosphodegron modulates temperature compensation, and shows functional temperature compensation heterogeneity within its residues.

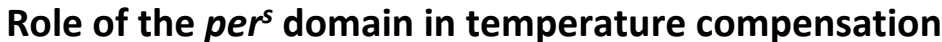

To further strengthen evidence for a role of phosphorylation at the PER phosphodegron in temperature compensation, we analyzed the impact of mutations in the $\mathrm{per}^{\mathrm{s}}$ domain (T/S583596). Indeed, this region also undergoes phosphorylation by DBT and NMO, and phosphorylation in the per $^{\text {s }}$ domain temporally precedes and inhibits phosphorylation at S47 (39). Therefore we tested point mutations in the per $^{s}$ domain for their role in temperature compensation.

We used previously described transgenic lines (39), in which the $\operatorname{per}^{0}$ mutation is rescued with per transgenes carrying various mutations in the $\mathrm{per}^{\mathrm{s}}$ domain. In addition, we included transgenic flies carrying S47A and S47D substitutions. Strikingly, the period length and overcompensation phenotypes observed with transgenes encoding PER S47A and S47D were similar to those observed with the corresponding mutants generated with CRISPR-CAS9 (Figure 1 and 2), confirming the critical role played by the $\$ 47$ residue in temperature compensation. Mutants in the per $^{s}$ domain exhibited shortened period length at $25^{\circ} \mathrm{C}$ (as described previously (39). They also showed consistently an undercompensation phenotype of about 1-1.5 hours, except for one S596A line (Figure 2B). This could be because of an insertional effect, since per transgenes were randomly inserted in the fly genome. It should 
be noted that the per ${ }^{T}$ and $p e r^{S}$ mutations have also been reported to be undercompensated (28-30). Taken together, our results and these previous reports show that the $\operatorname{per}^{S}$ domain is important for temperature compensation. Since $S$ to $A$ substitutions in this domain have opposite effects than S47A, and since phosphorylation of the per ${ }^{5}$ domain blocks S47 phosphorylation, it seems likely that the per $^{s}$ domain controls temperature compensation through 547 phosphorylation.

\section{S47A mutants show increased hyper-phosphorylated PER accumulation at warmer temperatures.}

To further understand the mechanism of temperature compensation, we examined PER protein cycling in control, S45A, and S47A mutants at $18^{\circ} \mathrm{C}$ and $29^{\circ} \mathrm{C}$ in head extracts. Under LD, control $\left(w^{1118}\right)$ flies showed rhythmic PER abundance and phosphorylation regardless of the temperature, albeit with significantly higher abundance at warmer temperatures (Figure $3 A, B$ and $C)$. This observation is in line with increased TIM abundance at warm temperature (40).

S47A also showed increased PER abundance at $29^{\circ} \mathrm{C}$ compared to $18^{\circ} \mathrm{C}$, but PER abundance and phosphorylation rhythms were perturbed at both temperature (Figure 3D, E F). Importantly, S47A mutants showed exaggerated temperature-sensitive accumulation of hyper-phosphorylated PER compared to controls at night (Figure 3G), but not of hypophosphorylated PER (Figure $3 \mathrm{H}$ ). Increased accumulation of hyper-phosphorylated PER is likely to result in longer repression phase thus lengthening the period length (41) at warmer temperatures. These results therefore reveal a temperature-dependent effect on PER phosphorylation and/or degradation of the S47A substitution, which could underlie the observed overcompensation phenotype.

S45A showed a milder phenotype than S47A, with patterns of phosphorylation similar to that of WT (Figure $3 \mathrm{I}, \mathrm{J}$ and $\mathrm{K}$ ). However, PER cycling amplitude at warmer temperatures was reduced and did not clear statistical significance. Phosphorylation rhythm amplitude were reduced in amplitude at both temperature as well.

\section{PER degradation is overcompensated as a result of the S47A substitution}


The increase in the accumulation of hyper-phosphorylated PER, observed at high temperature with the S47A substitution, could be the result of abnormal protein stability, possibly due to abnormal phosphorylation. We therefore turned to validated S2 cell culture assays (33) to determine the effect of temperature on PER phosphorylation and degradation kinetics. In these assays, recombinant DBT is expressed under the inducible metallothionine promoter, while PER is expressed constitutively. Since PER is phosphorylated on multiple sites by DBT and thus produce several phosphorylated isoforms with various mobility that are subjected to SLIMB-mediated proteasomal degradation, we quantified the disappearance of the hypophosphorylated PER after DBT induction to determine PER phosphorylation kinetics (Figure 4). Interestingly, phosphorylation kinetics of PER S47A was significantly slowed down 6 hours following DBT induction at low temperature (Figure 4A, C), while no significant effect was observed with either wild-type PER or S45A at this time point (Figure 4A, B and D). However, it seems unlikely that a slower phosphorylation kinetic at cold temperature would result in the shorter circadian behavior period observed in S47A mutants at $18^{\circ} \mathrm{C}$ compared to $29^{\circ} \mathrm{C}$

Since S47A caused increased hyper-phosphorylated PER accumulation at $29^{\circ} \mathrm{C}$, we also determined S47A's effects on PER stability. We again induced DBT and after 6 hours added cycloheximide to block protein synthesis. Wild-type PER was much more stable than S47A at $18^{\circ} \mathrm{C}$, while at $29^{\circ} \mathrm{C}$ there was no obvious difference (Figure $5 \mathrm{~B}$ and C). Strikingly, S47A's degradation kinetics was slowed down at higher temperature, while for WT, it was accelerated (Figure 5A, D, E). Thus, S47A results in a thermal overcompensation of PER degradation, which probably contribute to the excessive hyper-phosphorylated PER visible in flies expressing S47A, and thus causes overcompensation of circadian period (Figure 6). 


\section{Discussion:}

Phosphorylation events play an important role in the temperature compensation of the circadian clock across phyla $(8,9,11-13)$. In Drosophila, PER phosphorylation has been studied in exquisite detail, and interestingly three per mutations affecting two PER domains undergoing DBT phosphorylation are undercompensated. per ${ }^{S L I H}$ causes the most robust undercompensation phenotype, while $\operatorname{per}^{S}$ and $\operatorname{per}^{T}$ have milder effects $(25,28-30)$. We therefore systematically tested phosphorylated residues in both the $\mathrm{per}^{S}$ domain and the PER phosphodegron. Overall, our results point at a central role for residue S47, and modulatory roles for $\mathrm{S} 44, \mathrm{~S} 45$ and residue in the $\mathrm{per}^{\mathrm{S}}$ phosphorylation cluster.

Indeed, the S47A substitution resulted in a striking increase in temperature compensation. This suggests that phosphorylation at this residue is important for the compensation. Actually, we found that PER levels were exaggeratedly temperature-dependent in vivo, with a striking increase in hyper-phosphorylated PER levels at high temperature. Moreover, PER degradation induced by DBT phosphorylation was slowed down at high temperature, rather than accelerated by the S47A substitution in a Drosophila cell culture assay. These results thus suggest that $\mathrm{S} 47$ phosphorylation controls the kinetics of PER degradation in a temperaturedependent manner, protecting degradation from being overcompensated. This is further supported by the observation that mutations in the per $^{S}$ phosphocluster, which inhibits S47 phosphorylation, have opposite effects on temperature compensation: flies carrying these mutations are undercompensated. Unexpectedly though, the phosphomimetic substitution S47D did not produce an undercompensation phenotype. Rather, an overcompensation phenotype was observed. This could be explained by the substitution to Aspartate not properly mimicking the structure of a phosphorylated Serine, or that constantly mimicking S47 phosphorylation impacts thermal compensation of the clock through a different mechanism than the S47A substitution.

Another unexpected observation is that Serine to Alanine substitution at residues 44 and 45 have opposite effects on temperature compensation than S47A, even though all these substitutions lengthen circadian period. Residues of the phosphodegron are thus functionally heterogeneous when it comes to temperature compensation. Indeed, unlike S47A, S45A did not cause obvious change in temperature-dependent PER stability in vivo or in cell culture. It 
should be noted that unlike S47, S44 or S45 phosphorylation by DBT has not been unambiguously demonstrated (33). It remains thus possible that these residues are not phosphorylated and play a structural role in the phosphodegron, and this could explain functional diversity in temperature compensation between S44/45 and S47. Further studies are needed to elucidate S44 and S45's exact function in thermal compensation. Of note, the S45F substitution, which introduces a more bulky amino acid than S45A, caused a more severe phenotype that better mimicked the per ${ }^{S L I H}$ phenotype resulting from a S45Y substitution (Figure 1B, Table 1 (31)). This could indicate an important structural role for this amino acid, perhaps controlling specific protein-protein interactions not implicated in PER degradation.

Interestingly, our work, combined with previous work in mammals, converge to support the model that $\mathrm{CKI} \delta / \varepsilon$-mediated phosphorylation of PER proteins and their proteasomal degradation are critical for temperature compensation of the circadian clock. Surprisingly, however, there appear to be important mechanistic differences. Indeed, in Drosophila, mutation of the key Serine residue (S47) in the phosphodegron increases temperature compensation, while in mammals mutation of the homologous $\$ 478$ residue has the opposite effect (Figure 1B, 2B, Table 1 (12,13)). Intriguingly, in mammalian cell culture assays, phosphodegron mutation ( $\mathrm{S} 478 \mathrm{~A}$ in mammals) causes degradation kinetics to become essentially insensitive to temperature (12), while S47A degradation in flies is mildly overcompensated. However, in mammals, wild-type MPER2 degradation kinetics accelerates when temperature drops, while in flies we observed the opposite effect, perhaps because the phosphodegrons, though conserved, are located in different location in the PER and mPER2 proteins. This explains why mutations that both renders PER degradation less sensitive to temperature have opposite effect on temperature compensation.

Finally, it is important to point out that so far, any mutation or manipulation of phosphorylation of key circadian protein in any system only partially disrupts temperature compensation, or even increases it. It is therefore clear that multiple mechanisms are implicated, and recent results by Giesecke and collaborators (20) in flies for example point to an important role for nuclear export. It is therefore clear that temperature compensation is proving to be a complex, systemic process, as first proposed by Sweeney and Hastings in their seminal work on Dinoflagellates. 


\section{Material and Methods:}

Fly stocks: Fly stocks were maintained on standard cornmeal agar at $25^{\circ} \mathrm{C}$ under $12 \mathrm{hr}: 12 \mathrm{hr}$ light: dark (LD). The following available strains were used $w^{1118}$, yw , FM7a, w[1118]; PBac\{y[+mDint2]=vas-Cas9\}VK00027 (BL51324), y[1] M\{Act5C-Cas9.P.RFP-\}ZH-2A w[1118] DNAlig4[169] (BL58492). Transgenic flies used in Figure 2 were described previously $(33,39)$.

\section{CRISPR mutagenesis:}

Single stranded oligos (ssODNs) were injected into either BL51324 or BL58492 from Bloomington. The injected embryos, upon eclosion, were crossed with FM7a balancer flies. F1 progeny was screened with PCR and sequencing for the presence of the desired modifications.. All the resultant lines were backcrossed into the $w^{1118}$ genetic background. For lines generated from BL58492 (S45S\# I, S45S\# II, S45D \#122, \#67, S45A 98, S4445A\# 105, S45E, S45F \#51, S44D, S47D), we determined whether the Cas9 transgene located on the $X$ chromosome had been removed. S45E, S44D, S47D fly lines unexpectedly retained Cas9 even after multiple attempts to remove it. It seems however highly unlikely that Cas 9 could somehow interfere with temperature compensation, particularly since in both S45E and S44D temperature compensation was unaffected (Figure 1B).

\section{ssODN design:}

A conveniently located PAM site (TGG) was identified using CRISPR fly design. The guide RNA sequence chosen was - 5'AGCCACTGCTGCCGGAGGAGTGG. The guide RNAs were cloned into plasmid pCFD3 (Addgene, Cat no: 49410). Silent modifications (AGC-AGT) were introduced into the seed sequence to prevent re-cutting and for subsequent molecular screening.

\begin{tabular}{|l|l|}
\hline Genotype & ssODN sequence in $5^{\prime}$ to $3^{\prime}$ ' direction \\
\hline S45S & $\begin{array}{l}\text { ATCCATCCCCTTTCCAGTGGCAGCTCCAAATCCCGCCTGAGCGGCAGTCACTCCTCCGG } \\
\text { CAGCAGTGGCTATGGGGGCAAGCCCTCGACGCAGGCCAGCAGCAGCGACAT }\end{array}$ \\
\hline S45A & $\begin{array}{l}\text { ATCCATCCCCTTTCCAGTGGCAGCTCCAAATCCCGCCTGAGCGGCAGTCACTCCGCGG } \\
\text { GCAGCAGTGGCTATGGGGGCAAGCCCTCGACGCAGGCCAGCAGCAGCGACAT }\end{array}$ \\
\hline
\end{tabular}




\begin{tabular}{|c|c|}
\hline S45D & $\begin{array}{l}\text { ATCCATCCCCTTTCCAGTGGCAGCTCCAAATCCCGCCTGAGCGGCAGTCACTCCGACG } \\
\text { GCAGCAGTGGCTATGGGGGCAAGCCCTCGACGCAGGCCAGCAGCA\GCGACAT }\end{array}$ \\
\hline S45F & $\begin{array}{l}\text { ATCCATCCCCTTTCCAGTGGCAGCTCCAAATCCCGCCTGAGCGGCAGTCACTCCTTCGG } \\
\text { CAGCAGTGGCTATGGGGGCAAGCCCTCGACGCAGGCCAGCAGCAGCGACAT }\end{array}$ \\
\hline S45E & $\begin{array}{l}\text { ATCCATCCCCTTTCCAGTGGCAGCTCCAAATCCCGCCTGAGCGGCAGTCACTCCGAGG } \\
\text { GCAGCAGTGGCTATGGGGGCAAGCCCTCGACGCAGGCCAGCAGCAGCGACAT }\end{array}$ \\
\hline S44A & $\begin{array}{l}\text { ATCCATCCCCTTTCCAGTGGCAGCTCCAAATCCCGCCTGAGCGGCAGTCACGCGTCCG } \\
\text { GCAGCAGTGGCTATGGGGGCAAGCCCTCGACGCAGGCCAGCAGCAGCGACAT }\end{array}$ \\
\hline S44D & $\begin{array}{l}\text { ATCCATCCCCTTTCCAGTGGCAGCTCCAAATCCCGCCTGAGCGGCAGTCACGACTCCG } \\
\text { GCAGCAGTGGCTATGGGGGCAAGCCCTCGACGCAGGCCAGCAGCAGCGACAT }\end{array}$ \\
\hline S47A & $\begin{array}{l}\text { ATCCATCCCCTTTCCAGTGGCAGCTCCAAATCCCGCCTGAGCGGCAGTCACTCCTCCGG } \\
\text { CGCGAGTGGCTATGGGGGCAAGCCCTCGACGCAGGCCAGCAGCAGCGACAT }\end{array}$ \\
\hline S47D & $\begin{array}{l}\text { ATCCATCCCCTTTCCAGTGGCAGCTCCAAATCCCGCCTGAGCGGCAGTCACTCCTCCGG } \\
\text { CGACAGTGGCTATGGGGGCAAGCCCTCGACGCAGGCCAGCAGCAGCGACAT }\end{array}$ \\
\hline S44-45A & $\begin{array}{l}\text { ATCCATCCCCTTTCCAGTGGCAGCTCCAAATCCCGCCTGAGCGGCAGTCACGCGGCGG } \\
\text { GCTTCAGTGGCTATGGGGGCAAGCCCTCGACGCAGGCCAGCAGCAGCGACAT }\end{array}$ \\
\hline S45-47A & $\begin{array}{l}\text { ATCCATCCCCTTTCCAGTGGCAGCTCCAAATCCCGCCTGAGCGGCAGTCACTCCGCGG } \\
\text { GCGCGAGTGGCTATGGGGGCAAGCCCTCGACGCAGGCCAGCAGCAGCGACAT }\end{array}$ \\
\hline
\end{tabular}

Primers used:

Molecular screening for CRIPSR mutagenesis

\begin{tabular}{|l|l|l|l|}
\hline $\begin{array}{l}\text { Product } \\
\text { name }\end{array}$ & $\begin{array}{l}\text { Product } \\
\text { size }\end{array}$ & Forward primer(5'to3') & Reverse primer(5'to3') \\
\hline per & 1332 & GTTACCCCCATTCAAGGTCC & CCAAAACGGGCACAGATACT \\
\hline
\end{tabular}




\begin{tabular}{|l|l|l|l|}
\hline Act5-Cas9 & 771 & GATAAAAATCTGCCTAACG & CTTATGCCTTCCCATTACT \\
\hline $\begin{array}{l}\text { Vasa- } \\
\text { Cas9Set1 }\end{array}$ & 762 & GATAAGAACCTGCCCAAC & GCCCATCACTTTCACGA \\
\hline $\begin{array}{l}\text { Vasa- } \\
\text { Cas9Set2 }\end{array}$ & 761 & GCCTACCACGAGAAGTA & AGGATGGGCTTGATGAA \\
\hline lig4 & 784 & GCCAGCACGATCAAGTTC & GGTCTGCCAGCAGATTG \\
\hline
\end{tabular}

\section{Circadian locomotor activity monitoring and analysis:}

2-5 days old male flies were loaded into behavior tubes. Flies were entrained at the defined temperature for at least 3 days under 12:12 LD cycle and released into constant darkness (DD). Activity was recorded using the DAM (Drosophila Activity Monitoring) system (Trikinetics, Waltham, MA, USA) in 136-LL incubators (Percival). Behavior was analyzed and plotted using FaasX software (courtesy of F. Rouyer, Centre National de la Recherche Scientifique, Gif-surYvette, France). Rhythmicity was defined by following the criteria: power $>20$, width $>1.5$, using the $c 2$ periodogram analysis. 5 days of DD data were used to determine period. Period of all individual flies under various conditions can be found in supplemental file Figure 1-2 datasets.xls

\section{Statistical analysis:}

Whether difference in period at $29^{\circ} \mathrm{C}$ and $18^{\circ} \mathrm{C}$ were statistically significant was determined by using a two-way ANOVA followed by Sidak's multiple comparison tests. Since in figure 1 control flies showed a significant period shortening at warm temperatures compared to cold, we did factor this drift prior to run statistical test by subtracting the average period lengthening observed in S45SI, and S45SII (1.03hr) from the period observed at $18^{\circ} \mathrm{C}$ for each genotypes.

For graphical representation in figure 1 and 2 , mean difference between $29^{\circ} \mathrm{C}$ and $18^{\circ} \mathrm{C}$ were plotted on the $\mathrm{Y}$ axis and Standard Error (SE) of difference were used for error bars. Mean difference and SE of difference were obtained from the Two-way Anova statistics details is Prism. Western blots were analysed using Two-way ANOVA with Tukey's multiple comparison test. 


\section{Drosophila S2 cell culture and transfection:}

Drosophila S2 cells were maintained at $22^{\circ} \mathrm{C}$ in Schneider's Drosophila medium (Life Technologies (Carlsbad, CA)) supplemented with 10\% Fetal Bovine Serum (FBS) (VWR, Radnor, PA). Plasmids expressing pAc-per(WT)-V5, pAc-per(S45A)-V5, pAc-per(S47A)-V5 and pMT-dbtV5 were described in Ko et al. (2002) and Chiu et al. (2008). For all cell culture experiments, S2 cells were seeded at $1 \times 10^{6}$ cells $/ \mathrm{ml}$ in a 6-well plate and transfected using Effectene (Qiagen, Germantown, MD). For mobility shift assay (Figure 4), S2 cells were co-transfected with $0.2 \mu \mathrm{g}$ of pMT- $d b t-\mathrm{V} 5$ and $0.8 \mu \mathrm{g}$ of pAc-per(X)-V5, where $\mathrm{X}$ is either $\mathrm{WT}$, S45A or S47A. $d b t$ expression were induced with $500 \mu \mathrm{M} \mathrm{CuSO}_{4} 36$ hours after transfection and cells were moved into incubators with indicated temperature and cells were harvested at 0, 6, 12, 24 hours after induction. Proteins were analyzed by Western Blotting. For cycloheximide (CHX) chase experiment (Figure 5), S2 cells were co-transfected with $0.2 \mu \mathrm{g}$ of pMT- $d b t-\mathrm{V} 5$ and $0.8 \mu \mathrm{g}$ pAc-per(X)-V5, where $\mathrm{X}$ is either WT or S47A. $d b t$ expression were induced with $500 \mu \mathrm{M} \mathrm{CuSO}_{4}$ at 36 hours after transfection. 6 hours after DBT induction, CHX (Sigma) was added at a final concentration of $10 \mu \mathrm{g} / \mathrm{ml}$. Cells were harvested and lysed with EB2 (20mM HEPES pH 7.5, $100 \mathrm{mM} \mathrm{KCl}, 5 \%$ glycerol, 5mM EDTA, $1 \mathrm{mM}$ DTT, 0.1\% Triton X-100, 10 $\mu \mathrm{g} / \mathrm{ml}$ Aprotinin, $5 \mu \mathrm{g} / \mathrm{ml}$ Leupeptin, $1 \mu \mathrm{g} / \mathrm{ml}$ Pepstatin A, 0.5mM PMSF, 25mM NaF) at the indicated times. Proteins were analyzed by Western Blotting.

\section{Western blotting and antibodies:}

Protein extractions from Drosophila S2 cells and adult fly heads, western blotting, and image analysis was performed as previously described (Chiu et al., 2008; Cai et al., 2021). 2-5 day old flies were entrained to $12: 12$ light-dark cycle for 3 days at indicated temperature. On the $4^{\text {th }}$ day of LD, flies were collected at the indicated time points. $\sim 40$ fly heads for each time point were extracted with RBS buffer (20mM HEPES pH7.5, $50 \mathrm{mM} \mathrm{KCl,} 10 \%$ glycerol, $2 \mathrm{mM}$ EDTA, $1 \mathrm{mM}$ DTT, 1\% Triton X-100, 0.4\% NP-40, 10 $\mathrm{gg} / \mathrm{ml}$ Aprotinin, $5 \mu \mathrm{g} / \mathrm{ml}$ Leupeptin, $1 \mu \mathrm{g} / \mathrm{ml}$ Pepstatin, 0.5mM PMSF, 25mM NaF). Protein concentration was measured using Pierce Coomassie Plus Assay Reagents (Thermo Fisher Scientific). 2X SDS sample buffer was added and the mixture boiled at $95^{\circ} \mathrm{C}$ for 5 minutes. Equal amounts of proteins were resolved by polyacrylamide-SDS gel electrophoresis (PAGE) and transferred to nitrocellulose membrane (Bio-Rad, Hercules, CA) using Semi-Dry Transfer Cell (Bio-Rad). Membranes were stained with 
Ponceau S solution ( $0.1 \%$ Ponceau S, $5 \%$ acetic acid) to stain total proteins as loading control. Membranes were then incubated in 5\% Blocking Buffer (Bio-Rad) for 40 minutes, incubated with primary antibodies for 16-20 hours. Blots were then washed with 1X TBST for 1 hour, incubated with secondary antibodies for 1 hour, washed again prior to treatment of chemiluminescence Clarity ECL reagent (Bio-Rad). The following percentage of polyacrylamide-SDS gel were used: $6 \%$ for PER; $10 \%$ for DBT and HSP70.

Primary antibodies: $\alpha$-V5 (Thermo Fisher Scientific) at 1:3000 for PER-V5 and DBT-V5, $\alpha-P E R$ (GP5620; RRID:AB_2747405) at 1:2000 for PER, $\alpha-H S P 70$ at 1:10000 for HSP70. Secondary antibodies conjugated with HRP were added as follows: $\alpha$-mouse IgG (Sigma) at 1:2000 for $\alpha$ V5 detection, 1:10000 for $\alpha$-HSP70 detection, $\alpha$-guinea pig IgG (Sigma) at 1:1000 for $\alpha$-PER detection. All quantifications are included in supplemental file Figure 3-5-datasets.xls

\section{Acknowledgments:}

We thank VInh Phan for technical support, and members of the Emery, Weaver and Anaclet labs for helpful discussions. This work was supported by MIRA award 1R35GM118087 from the National Institute of General Medicine Sciences (NIGMS) to PE, and R01 DK124068 from the National Institute of Diabetes and Digestive and Kidney Diseases (NIDDK) to JCC. 


\section{Bibliography:}

1. Dunlap JC. Molecular Bases for Circadian Clocks. Cell. 1999;96(2):271-90.

2. Hardin PE. Molecular genetic analysis of circadian timekeeping in Drosophila. Advances in Genetics. 2011;74:141-173.

3. Lam VH, Li YH, Liu X, Murphy KA, Diehl JS, Kwok RS, et al. CK1 $\alpha$ Collaborates with DOUBLETIME to Regulate PERIOD Function in the Drosophila Circadian Clock. J Neurosci. 2018;38(50):10631-43.

4. Tataroglu O, Emery P. The molecular ticks of the Drosophila circadian clock. Curr Opin Insect Sci. 2015;7:51-7.

5. Pittendrigh CS. Circadian rhythms and the circadian organization of living systems. Cold Spring Harb Symp Quant Biol. 1960;25:159-84.

6. Pittendrigh CS. on Temperature Independence in the Clock System Controlling Emergence Time in Drosophila. Proc Natl Acad Sci. 1954;40(10):1018-29.

7. Hastings JW, Sweeney BM. on the Mechanism of Temperature Independence in a Biological Clock. Proc Natl Acad Sci. 1957;43(9):804-11.

8. Mehra A, Shi M, Baker CL, Colot H V., Loros JJ, Dunlap JC. A Role for Casein Kinase 2 in the Mechanism Underlying Circadian Temperature Compensation. Cell. 2009;137(4):749-60.

9. Portolé S, Má P. The Functional Interplay between Protein Kinase CK2 and CCA1 Transcriptional Activity Is Essential for Clock Temperature Compensation in Arabidopsis. 2010;6(11):1001201.

10. Isojima Y, Nakajima M, Ukai H, Fujishima H, Yamada RG, Masumoto KH, et al. CKIE/ $\delta$ dependent phosphorylation is a temperature-insensitive, period-determining process in the mammalian circadian clock. Vol. 106, Proceedings of the National Academy of Sciences of the United States of America. 2009;106(37):15744-749

11. Tosini G, Menaker M. The tau mutation affects temperaturecompensation of hamster retinal circadian oscillators. 1998;9(6):1001-5.

12. Zhou M, Kim JK, Eng GWL, Forger DB, Virshup DM. A Period2 Phosphoswitch Regulates and Temperature Compensates Circadian Period. Mol Cell. 2015;60(1):7788.

13. Masuda S, Narasimamurthy R, Yoshitane H, Kim JK, Fukada Y, Virshup DM. Mutation of a PER2 phosphodegron perturbs the circadian phosphoswitch. Proc Natl Acad Sci U S A. 2020;117(20):10888.

14. Eide EJ, Woolf MF, Kang H, Woolf $P$, Hurst W, Camacho F, et al. Control of mammalian circadian rhythm by CKlepsilon-regulated proteasome-mediated PER2 degradation. Mol Cell Biol. 2005;25(7):2795-807. 
15. Reischl S, Vanselow K, Westermark PO, Thierfelder N, Maier B, Herzel H, et al. BetaTrCP1-mediated degradation of PERIOD2 is essential for circadian dynamics. J Biol Rhythms. 2007;22(5):375-86.

16. Toh KL, Jones CR, He Y, Eide EJ, Hinz WA, Virshup DM, et al. An hPer2 phosphorylation site mutation in familial advanced sleep phase syndrome. Science.

2001;291(5506):1040-3.

17. Shanware NP, Hutchinson JA, Kim SH, Zhan L, Bowler MJ, Tibbetts RS. Casein kinase 1dependent phosphorylation of familial advanced sleep phase syndrome-associated residues controls PERIOD 2 stability. J Biol Chem. 2011;286(14):12766-74.

18. Narasimamurthy R, Hunt SR, Lu Y, Fustin JM, Okamura H, Partch CL, et al. CK1ס/e protein kinase primes the PER2 circadian phosphoswitch. Proc Natl Acad Sci U S A . 2018;115(23):5986-91.

19. Hansen LL, van den Burg HA, van Ooijen G. Sumoylation Contributes to Timekeeping and Temperature Compensation of the Plant Circadian Clock. J Biol Rhythms . 2017;32(6):560-9.

20. Giesecke A, Johnstone PS, Lamaze A, Landskron J, Atay E, Chen K-F, et al. Nuclear Export of Drosophila PERIOD contributes to temperature compensation of the circadian clock. bioRxiv. 2021;2021.10.25.465663.

21. Singh S, Giesecke A, Damulewicz M, Fexova S, Mazzotta GM, Stanewsky R, et al. New Drosophila Circadian Clock Mutants Affecting Temperature Compensation Induced by Targeted Mutagenesis of Timeless. Front Physiol. 2019;10

22. Gekakis N, Saez L, Delahaye-Brown AM, Myers MP, Sehgal A, Young MW, et al. Isolation of timeless by PER protein interaction: Defective interaction between timeless protein and long-period mutant PERL. Science. 1995;270(5237):811-5.

23. Huang ZJ, Curtin KD, Rosbash M. PER protein interactions and temperature compensation of a circadian clock in Drosophila. Science. 1995;267(5201):1169-72.

24. Huang ZJ, Edery I, Rosbash M. PAS is a dimerization domain common to Drosophila period and several transcription factors. Nature. 1993;364(6434):259-62.

25. Ewer J, Hamblen-Coyle M, Rosbash M, Hall JC. Requirement for period gene expression in the adult and not during development for locomotor activity rhythms of imaginal drosophila melanogaster. J Neurogenet. 1990;7(1):31-73.

26. Sawyer LA, Hennessy JM, Peixoto AA, Rosato E, Parkinson H, Costa R, et al. Natural variation in a Drosophila clock gene and temperature compensation. Science. 1997;278(5346):2117-20.

27. Peixoto AA, Hennessy JM, Townson I, Hasan G, Rosbash M, Costa R, et al. Molecular coevolution within a Drosophila clock gene. Proc Natl Acad Sci U S A. 1998;95(8):4475-80.

28. Konopka RJ, Hamblen-Coyle MJ, Jamison CF, Hall JC. An Ultrashort Clock Mutation at 
the period Locus of Drosophila melanogaster That Reveals Some New Features of the Fly's Circadian System. J Biol Rhythms. 1994;9:189-216.

29. Bao S, Rihel J, Bjes E, Fan JY, Price JL. The Drosophila double-times mutation delays the nuclear accumulation of period protein and affects the feedback regulation of period mRNA. J Neurosci. 2001;21(18):7117-26.

30. Rothenfluh A, Abodeely M, Price JL, Young MW. Isolation and analysis of six timeless alleles that cause short- or long-period circadian rhythms in Drosophila. Genetics. 2000;156(2):665-75.

31. Hamblen MJ, White NE, Emery PTJ, Kaiser K, Hall JC. Molecular and behavioral analysis of four period mutants in Drosophila melanogaster encompassing extreme short, novel long, and unorthodox arrhythmic types. Genetics. 1998;149(1):165-78.

32. Matsumoto A, Tomioka K, Chiba Y, Tanimura T. tim rit Lengthens Circadian Period in a Temperature-Dependent Manner through Suppression of PERIOD Protein Cycling and Nuclear Localization . Mol Cell Biol. 1999;19(6):4343-54.

33. Chiu JC, Vanselow JT, Kramer A, Edery I. The phospho-occupancy of an atypical SLIMB-binding site on PERIOD that is phosphorylated by DOUBLETIME controls the pace of the clock. Genes Dev. 2008;22(13):1758-72.

34. Chiu JC, Ko HW, Edery I. NEMO/NLK phosphorylates PERIOD to initiate a time-delay phosphorylation circuit that sets circadian clock speed. Cell. 2011;145(3):357-70.

35. Kloss B, Price JL, Saez L, Blau J, Rothenfluh A, Wesley CS, et al. The Drosophila clock gene double-time encodes a protein closely related to human casein kinase l$\varepsilon$. Cell. 1998;94(1):97-107.

36. Price JL, Blau J, Rothenfluh A, Abodeely M, Kloss B, Young MW. double-time is a novel Drosophila clock gene that regulates PERIOD protein accumulation. Cell. 1998;94(1):83-95.

37. Grima B, Lamouroux A, Chélot E, Papin C, Limbourg-Bouchon B, Rouyer F. The F-box protein Slimb controls the levels of clock proteins Period and Timeless. Nature. 2002;420(6912):178-82.

38. Ko HW, Jiang J, Edery I. Role for Slimb in the degradation of Drosophila Period protein phosphorylated by Doubletime. Nature. 2002;420(6916):673-8.

39. Chiu JC, Ko HW, Edery I. NEMO/NLK phosphorylates PERIOD to initiate a time-delay phosphorylation circuit that sets circadian clock speed. Cell. 2011;145(3):357-70.

40. Kidd PB, Young MW, Siggia ED. Temperature compensation and temperature sensation in the circadian clock. Proc Natl Acad Sci U S A. 2015;112(46):E6284-92.

41. Kivimäe S, Saez L, Young MW. Activating PER repressor through a DBT-directed phosphorylation switch. PLoS Biol. 2008;6(7):1570-83. 


\begin{tabular}{|c|c|c|c|c|c|c|c|c|c|c|c|c|}
\hline \multirow[b]{2}{*}{ Degree Celsius } & \multicolumn{4}{|c|}{ Period (h) } & \multicolumn{4}{|c|}{ \% Rhythmicity } & \multicolumn{4}{|c|}{ \# Rhythmic flies } \\
\hline & 29 & 25 & 20 & 18 & 29 & 25 & 20 & 18 & 29 & 25 & 20 & 18 \\
\hline $\begin{array}{l}\text { Phosphodegron cluster } \\
\text { W1118 }\end{array}$ & 23.6 & 24.5 & 24.3 & 24.5 & 87.5 & 86.9 & 53.8 & 40.0 & 93 & 53 & 42 & 32 \\
\hline S45S (1) & 23.8 & 25.0 & 24.8 & 25.1 & 80.7 & 95.7 & 76.6 & 76.6 & 71 & 44 & 85 & 36 \\
\hline S45S (2) & 23.9 & 24.9 & 24.8 & 24.7 & 83.3 & 95.3 & 61.0 & 84.7 & 60 & 61 & 64 & 50 \\
\hline S45F (51) & 26.6 & 27.7 & 28.7 & 29.2 & 62.9 & 83.9 & 71.3 & 87.1 & 34 & 26 & 67 & 54 \\
\hline S45F(111) & 26.4 & 27.3 & 28.2 & 28.7 & 60.0 & 53.3 & 65.8 & 37.5 & 18 & 8 & 25 & 12 \\
\hline$S 45 F(115)$ & 26.2 & 27.5 & 28.3 & 28.7 & 62.9 & 90.9 & 77.8 & 67.7 & 17 & 20 & 35 & 21 \\
\hline S45A (73) & 26.3 & 27.2 & 28.0 & 28.5 & 76.6 & 93.5 & 82.1 & 87.5 & 36 & 29 & 78 & 56 \\
\hline S45A (5) & 26.0 & 26.7 & 27.2 & 28.1 & 80.4 & 62.5 & 36.6 & 60.0 & 37 & 10 & 34 & 18 \\
\hline S45A (90) & 26.3 & 26.8 & 27.8 & 28.4 & 65.0 & 93.8 & 75.4 & 70.0 & 26 & 15 & 46 & 21 \\
\hline S45D (67) & 24.1 & 24.9 & 24.8 & 24.9 & 84.4 & 100.0 & 76.0 & 95.1 & 38 & 32 & 73 & 58 \\
\hline S45D (122) & 24.0 & 24.9 & 24.8 & 24.8 & 84.1 & 95.1 & 80.0 & 91.1 & 53 & 58 & 88 & 51 \\
\hline S45E & 23.9 & 24.7 & 24.2 & 24.9 & 87.2 & 93.3 & 44.2 & 35.4 & 41 & 43 & 19 & 17 \\
\hline S44A (29) & 24.9 & 25.8 & 25.7 & 26.8 & 88.6 & 97.5 & 82.1 & 73.9 & 70 & 39 & 69 & 34 \\
\hline S44A (20) & 24.9 & 26.0 & 26.6 & 26.9 & 77.1 & 91.3 & 84.8 & 89.1 & 37 & 21 & 56 & 41 \\
\hline S44A (62) & 24.7 & 24.2 & ND & 27.0 & 76.7 & 75.0 & ND & 55.0 & 33 & 14 & ND & 33 \\
\hline S44D & 25.6 & 26.6 & 26.9 & 26.7 & 62.5 & 90.7 & 66.7 & 35.7 & 25 & 39 & 29 & 20 \\
\hline S47A (19) & 32.1 & 31.3 & 31.0 & 31.2 & 28.8 & 53.8 & 46.9 & 52.1 & 17 & 44 & 15 & 49 \\
\hline S47A (21) & 33.5 & 31.5 & 31.5 & 31.3 & 38.3 & 44.9 & 15.6 & 58.8 & 18 & 48 & 5 & 47 \\
\hline S47A (35) & 33.0 & 31.5 & 31.3 & 31.4 & 23.3 & 72.9 & 37.5 & 47.7 & 17 & 70 & 12 & 53 \\
\hline S47D & 22.5 & 22.6 & 21.7 & 21.1 & 88.5 & 97.9 & 93.6 & 72.7 & 46 & 47 & 44 & 40 \\
\hline S4445A (105) & 34.4 & 35.9 & 35.3 & 38.0 & 56.3 & 32.3 & 35.6 & 6.3 & 9 & 10 & 16 & 1 \\
\hline S4445A (32) & 34.0 & AR & 35.6 & AR & 19.4 & 0.0 & 4.2 & 0.0 & 6 & 0 & 2 & 0 \\
\hline S4445A (60) & 34.1 & AR & 32.2 & AR & 59.4 & 0.0 & 25.4 & 0.0 & 19 & 0 & 16 & 0 \\
\hline S4547A (1) & AR & AR & AR & AR & 0.0 & 0.0 & 0.0 & 0.0 & & & & \\
\hline S4547A (30) & AR & AR & AR & AR & 0.0 & 0.0 & 0.0 & 0.0 & & & & \\
\hline S4547A (57) & AR & $A R$ & AR & AR & 0.0 & 0.0 & 0.0 & 0.0 & & & & \\
\hline S4547A (84) & AR & AR & $A R$ & $A R$ & 0.0 & 0.0 & 0.0 & 0.0 & & & & \\
\hline S4547A (90) & AR & AR & AR & AR & 0.0 & 0.0 & 0.0 & 0.0 & & & & \\
\hline \multicolumn{13}{|l|}{ pers cluster } \\
\hline $13.2 \mathrm{WT}$ & 23.8 & 24.2 & 23.9 & 23.8 & 89.3 & 76.6 & 23.3 & 32.9 & 75 & 37 & 15 & 27 \\
\hline S47A (M28-F1) & 32.7 & 30.5 & 30.0 & 29.9 & 48.3 & 44.7 & 9.9 & 27.2 & 43 & 21 & 9 & 28 \\
\hline S47A (F15-F1) & 32.6 & 31.6 & 31.4 & 29.8 & 96.8 & 95.8 & 65.6 & 50.8 & 61 & 46 & 43 & 32 \\
\hline S47D (M7-M2) & 21.7 & 21.8 & 21.5 & 21.0 & 69.6 & 80.4 & 68.1 & 44.8 & 48 & 37 & 47 & 23 \\
\hline S596A (F1-F1) & 15.9 & 15.2 & 15.9 & 16.3 & 75.0 & 91.5 & 18.8 & 8.5 & 33 & 43 & 9 & 4 \\
\hline S596A (M16-F1) & 15.7 & 15.5 & 16.8 & 17.2 & 68.1 & 93.8 & 95.7 & 81.6 & 32 & 45 & 44 & 62 \\
\hline TS583-596A (F30-M1) & 16.9 & 17.4 & 17.7 & 17.8 & 22.9 & 72.3 & 60.4 & 67.3 & 11 & 34 & 29 & 37 \\
\hline TS583-596A (M3-F1) & 16.2 & 16.3 & 17.0 & 17.3 & 61.7 & 80.9 & 82.6 & 86.8 & 29 & 38 & 24 & 79 \\
\hline TS583-596D (F2-F1) & 15.3 & 15.0 & 15.8 & 16.0 & 55.3 & 91.5 & 45.8 & 48.6 & 26 & 43 & 22 & 41 \\
\hline TS583-596D (F2-M1) & 15.1 & 15.2 & 15.7 & 16.0 & 68.8 & 78.6 & 69.0 & 60.0 & 22 & 22 & 20 & 37 \\
\hline S589A (M8-F1) & 19.4 & 19.6 & 21.0 & 20.7 & 95.1 & 97.9 & 97.9 & 76.7 & 39 & 46 & 46 & 56 \\
\hline
\end{tabular}

Table I: Circadian behavior of control and per mutant flies under different temperatures.

Mutations in the phosphoclusters were generated by CRISPR/Cas9-mediated genome editing. Amino acid substituions are indicated in the left column. $w^{1118}$ is a wild-type strain. For the $\mathrm{per}^{\mathrm{S}}$ phosphocluster, per $^{0}$ flies were rescued with various transgenes. 13.2WT carries a wild-type per genomic region, while the other transgenes carry the indicated subsitutions. $A R=$ arrhythmic ND = not done 


\section{Figure legends:}

Figure 1: Differential modulation of temperature compensation by three Serine residues in the PER phosphodegron

A) Schematic of PER protein depicting the relative positions of amino acids modified in various mutants affecting temperature compensation in Drosophila. Mutants above the PER schematic are overcompensated while mutants depicted below are undercompensated mutants.

The magnified region of PER shows residues of the APER phosphodegron and the various substitutions (Serine to Alanine, Aspartate or Glutamate) we generated using CRISPR/CAS9. Solid P within black circle, shows that S47 position undergoes phosphorylation. Grey circles indicate potential additional phosphorylation sites at S44 and S45 (33).

B) Graphical representation of the difference in period values of various mutants of dPER phosphodegron at $29^{\circ} \mathrm{C}$ and $18^{\circ} \mathrm{C}$, relative to controls. $\Delta$ Period values for each genotype were offset by the average difference in period observed at $29^{\circ} \mathrm{C}$ and $18^{\circ} \mathrm{C}$ in S45SI and S45SII control lines, so that overcompensation or undercompensation compared to control is clearly represented. The color scheme denotes period of each mutant line at $25^{\circ} \mathrm{C}$. Interestingly, S44A, S45A and S47A all produce long period phenotype at $25^{\circ} \mathrm{C}$. However, the directionality of their temperature compensation phenotypes is opposite between S44A/S45A and S47A. S44A and S45A produce significant undercompensation while S47A and S47D shows overcompensation. Statistical significance was calculated using two-way ANOVA with Sidak's multiple comparison test.

\section{Figure 2: the $\operatorname{per}^{S}$ phosphocluster impacts temperature compensation}

A) PER schematic depicting the relative position of various mutants affecting temperature compensation, as shown in figure $1 \mathrm{~A}$. The magnified region shows key Serine residues of the per $^{S}$ phosphocluster and the various substitutions that were tested. Solid P within black circle at 589 and 596 shows sites of DBT and NMO mediated phosphorylation, respectively. Dotted grey circles show predicted phosphorylation sites at S585 and S583. Underlines at S589 and G593 denote positions of per $^{S}$ and $p e r^{T}$ mutant, respectively. 
B) Graphical representation of the difference in period of various mutants of the $p e r^{s}$ domain at $29^{\circ} \mathrm{C}$ and $18^{\circ} \mathrm{C}$. The color scheme denotes period of each mutant line at $25^{\circ} \mathrm{C}$.

All mutants in the $p e r^{s}$ domain produce short period phenotype at $25^{\circ} \mathrm{C}$. Most show significant undercompensation. Transgenic lines with S47A recapitulated our observations with CRISPRCas9 genome editing (figure 1). Transgenic S47D showed a weaker phenotype than genome edited S47D, just short of statistical significance $(P=0.07)$. Statistical significance was calculated using two-way ANOVA with Sidak's multiple comparison test.

Figure 3: S47A mutation causes hyper-phosphorylated PER to accumulate preferentially at high temperature

A, D and I) Representative western blots probing head extracts from flies entrained to LD at $18^{\circ} \mathrm{C}$ and $29^{\circ} \mathrm{C}$. PER abundance was monitored throughout the day at the indicated time points. Respective genotypes are represented at the top of each gel. Brackets indicate hyperand hypo-phosphorylated PER isoforms. Two different exposures of the same blot are shown. Ponceau S was used as a loading control and for normalization.

B-F and J-K) Graphs show PER abundance and phosphorylation quantifications. In control flies, both PER phosphorylation (B) and abundance (C) were scored as statistically rhythmic at $29^{\circ} \mathrm{C}$ and $18^{\circ} \mathrm{C}$, using the JTK_CYCLE. For phosphorylation: $p<0.05$, phase $=2$ at $18^{\circ} \mathrm{C}$ and $p<0.001$, phase $=2$ at $29^{\circ} \mathrm{C}$. For abundance: $p<0.05$, phase $=18$ at $18^{\circ} \mathrm{C}$ and $p<0.001$, phase $=20$ at $29^{\circ} \mathrm{C}$. For S47A, neither phosphorylation (E) nor abundance (F) were scored as statistically rhythmic, although PER levels were reproducibly lower at ZT4. For S45A, only PER abundance at $18^{\circ} \mathrm{C}$ scored as statistically rhythmic. $p<0.05$, phase $=18$. Red lines represent data at $29^{\circ} \mathrm{C}$ and blue lines represent data at $18^{\circ} \mathrm{C} .{ }^{*} p<0.05, * * p<0.01, * * * p<0.001$, two-way ANOVA followed by Sidak's multiple comparison to test for time and temperature-dependent differences $(n=3$ biological replicates).

G-H) Ratio of PER levels measured at $29^{\circ} \mathrm{C}$ vs $18^{\circ} \mathrm{C}$. G shows the ratio for hyperphosphorylated PER, and $\mathrm{H}$ for hypo-phosphorylated PER. S47A accumulates more hyperphosphorylated PER during the night than control flies at warm temperatures.

\section{Figure 4: S47A phosphorylation kinetics is slowed down at cold temperature}

Representative western blots probing cell extracts from S2 cells expressing DBT and either wild-type (WT) PER, S47A or S45A. Cells were incubated at the indicated temperatures, and 
collected at the indicated time points after DBT induction. Top panel shows PER immunoblotting, bottom panel shows Ponceau S staining, used as loading control and for normalization.

$B, C$ and D) Western Blot quantifications. PER signal shown at time point 0 is used as a reference to classify PER isoforms as hypo-phosphorylated. Graphs representing the relative amount of hypo-phosphorylated PER at 18,25 and $29^{\circ} \mathrm{C}$ for the respective genotypes at different time points after DBT induction. S47A shows increased hypo-phosphorylated PER 6h after DBT induction. ${ }^{*}<<0.05$, two-way ANOVA followed by Sidak's multiple comparison to test for time and temperature-dependent differences ( $n=3$ biological replicates).

\section{Figure 5: S47A degradation kinetics is excessively temperature-compensated}

A) Representative western blots probing cell extracts from S2 cells expressing DBT and either wild-type (WT) PER, S47A or S45A. Cells were collected at the indicated time points after cycloheximide addition at the indicated temperatures. Top panel shows PER immunoblotting, bottom panel shows Ponceau S staining, used as loading control and for normalization.

B-E) Western Blot quantifications. All experiments were performed with three independent replicates. ${ }^{*} p<0.05, * * p<0.01, * * * p<0.001$, two-way ANOVA followed by Sidak's multiple comparison to test for time and temperature-dependent differences. In B and C, WT and S47A mutants are compared to each other at the indicated temperature. $D$ and $E$ show the same data, but degradation kinetics of WT and S47A are compared between high and low temperatures.

\section{Figure 6: Model for the role of PER phosphoclusters in temperature compensation}

A) The PER phosphodegron (yellow) and $p e r^{s}$ phosphocluster (green) modulate temperature compensation. Mutations of different residues of the PER phosphodegron have opposite effects on temperature compensation. S44A and S45A cause undercompensation while S47A results in overcompensation of circadian period. Mutations in the $\operatorname{per}^{s}$ phosphocluster cause undercompensation, consistent with its inhibitory role on S47 phosphorylation (red arrow). Black ovals around 47, 589, and 596 show DBT and NMO mediated phosphorylation sites on PER. Grey ovals around 44, 45, 583, and 585 depict putative additional phosphorylation sites. B) Mechanism of S47 action on temperature compensation. In wild-type flies, S47 phosphorylation causes PER degradation to speed up as temperature increases. Hyper- and 
hypo-phosphorylated isoforms are in balance and the clock is properly temperaturecompensated. In S47A mutant flies, PER degradation is overcompensated and thus slows down at high temperature, leading to excessive accumulation of hyperphosphorylated isoforms. The clock is then overcompensated. Violet ovals represent PER protein, Halo around violet ovals show hyper-phosphorylated PER. 

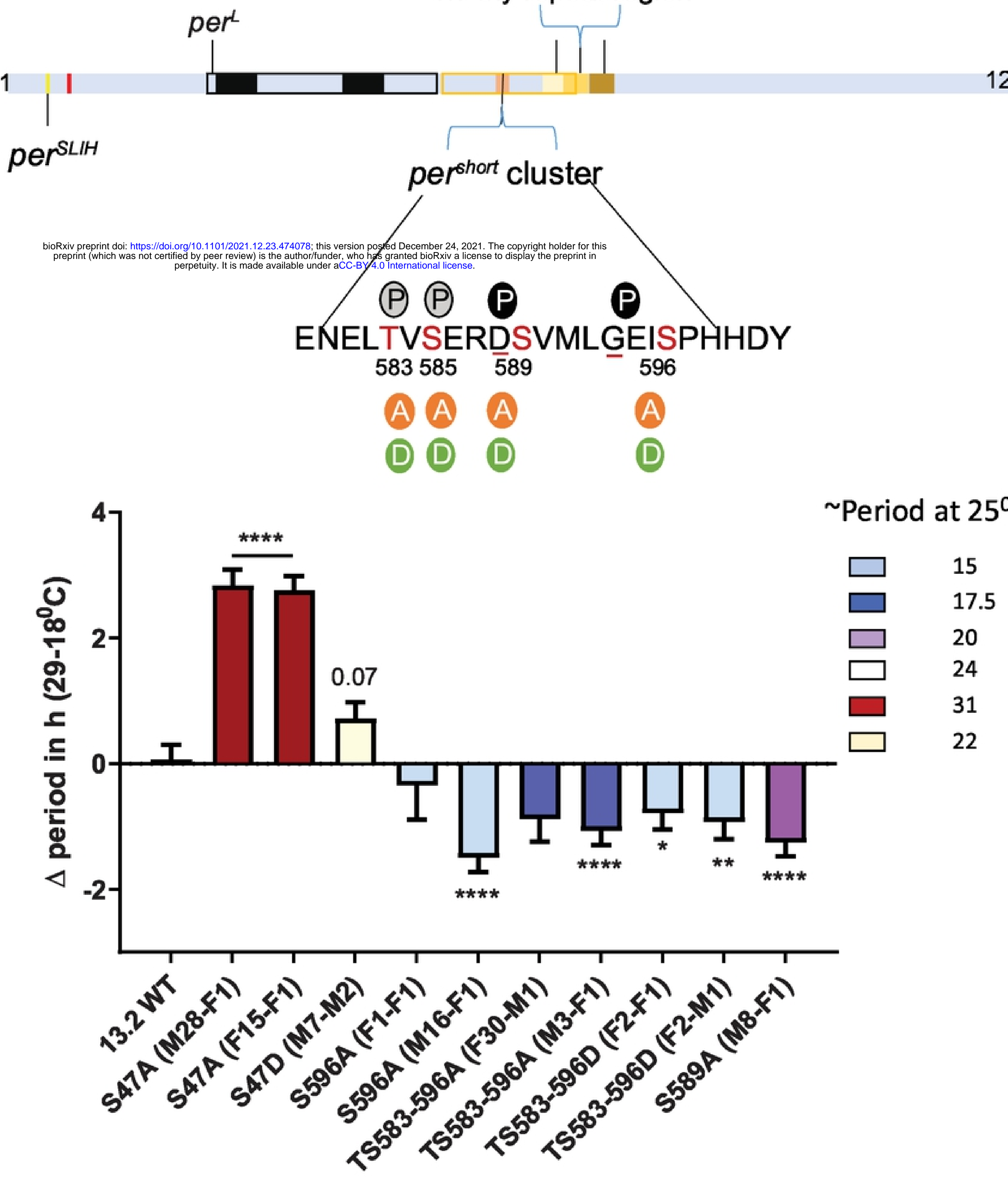

Figure 2 
Figure 1

A
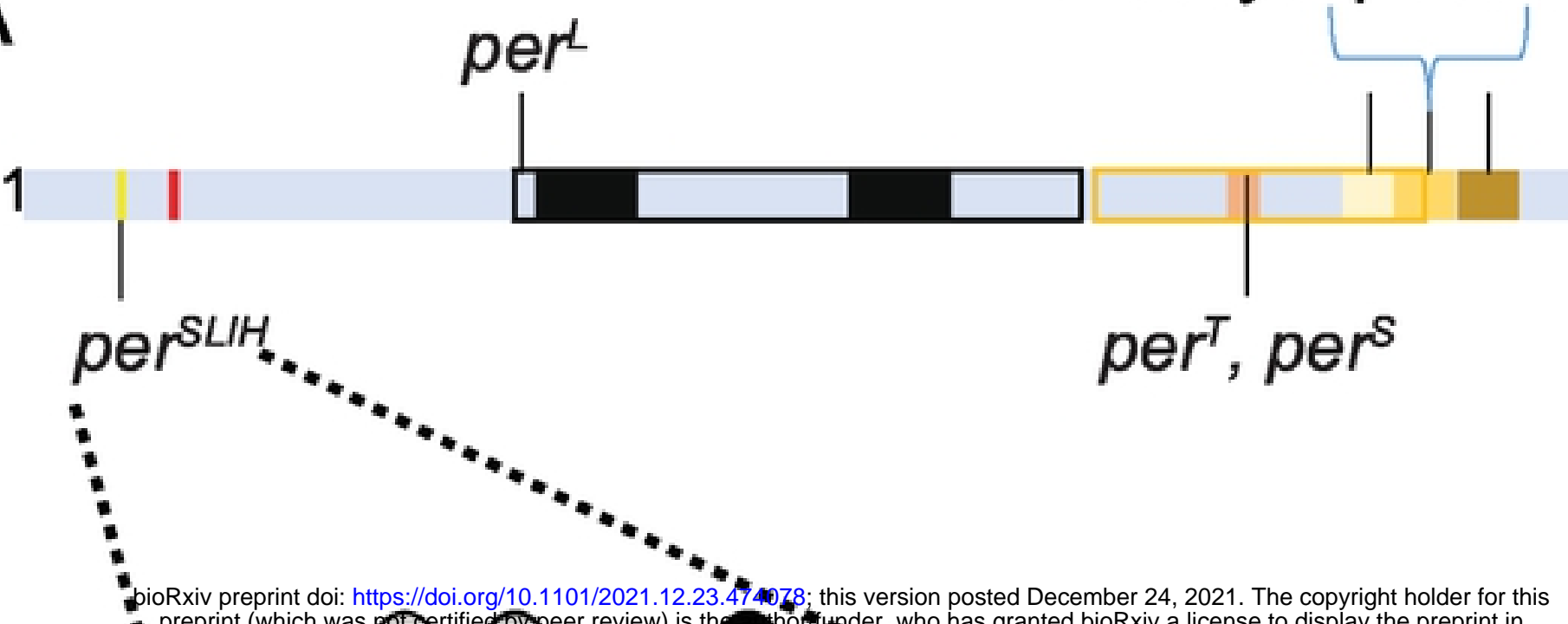

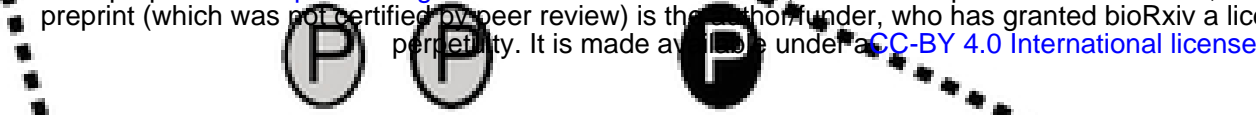

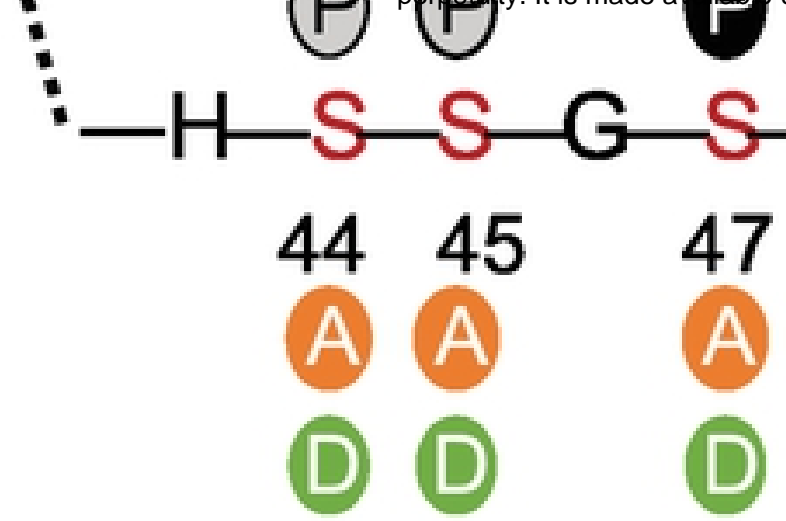

PER phosphodegron

Period at $25^{\circ} \mathrm{C}(\mathrm{h})$

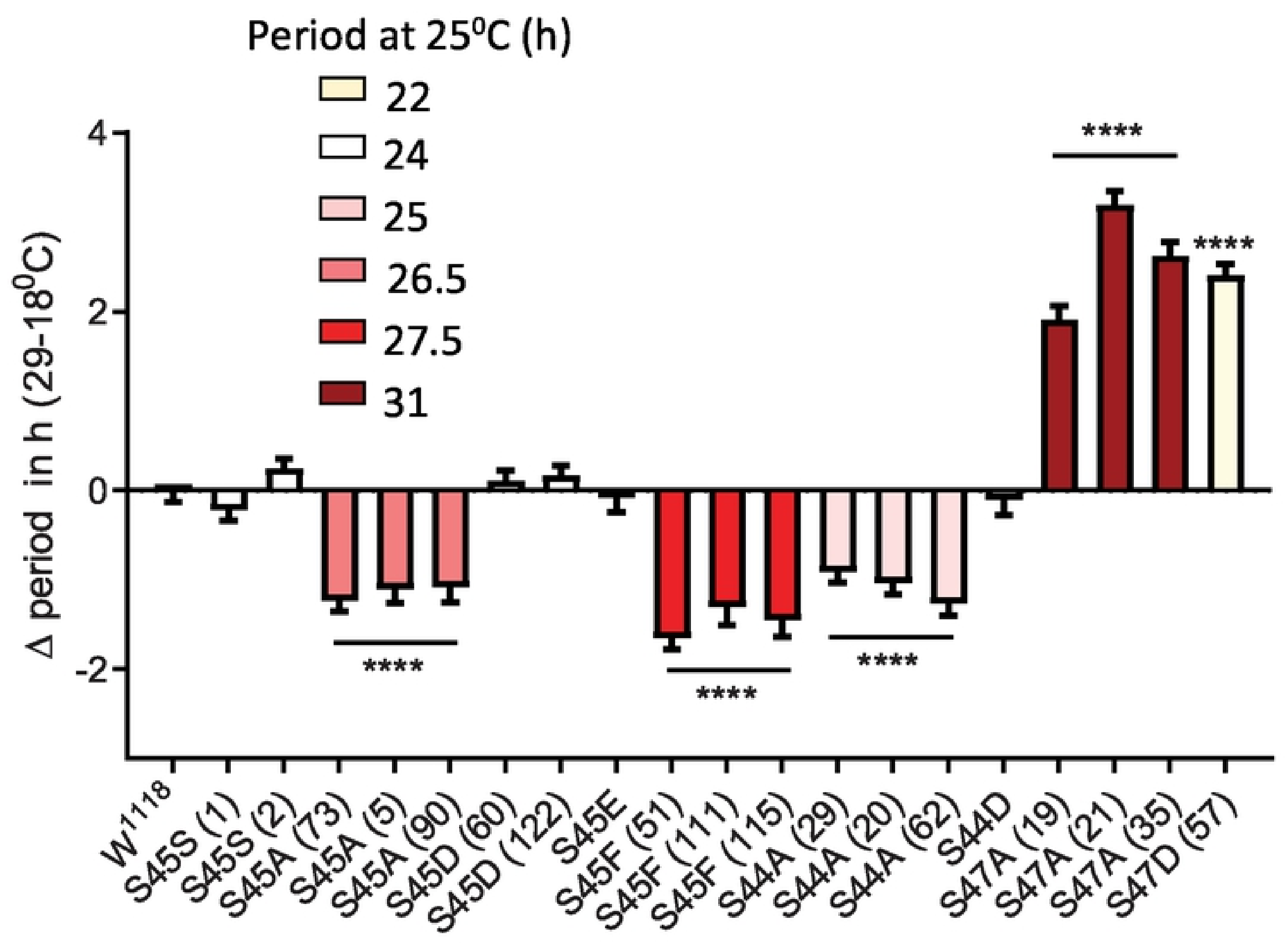

Figure 1 
Figure 3

A

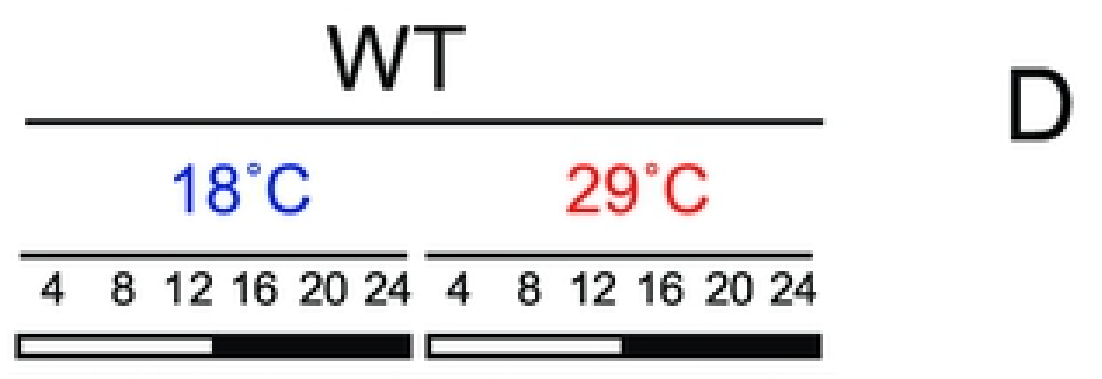

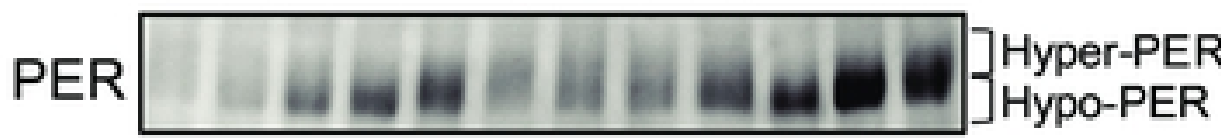

PER

(longer exposure)

bioRxiv preprint doi: https://doi.org/10.1101/2021.12.23.474078; this version posted December 24, 2021. The copyright h(фtalargerthioxposure)

Pon ceau $\mathrm{S}$ perpetuity. It is made available under aCC-BY 4.0 International license

B

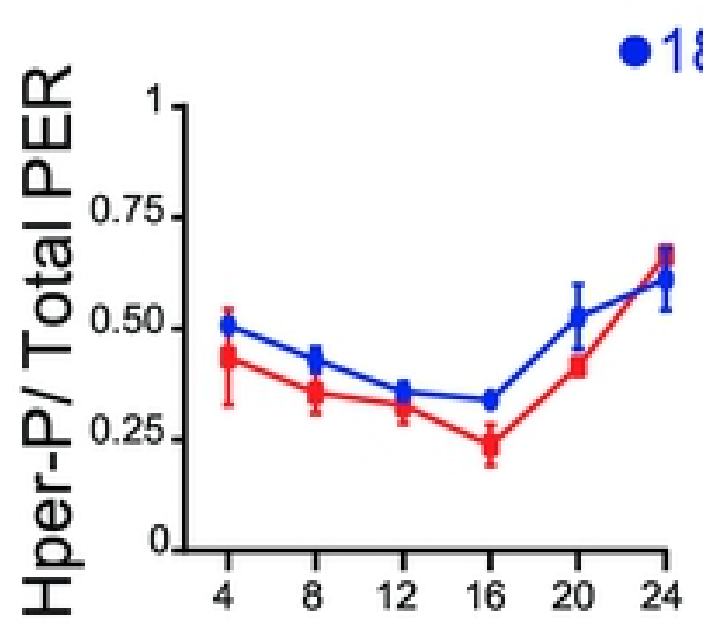

$G$

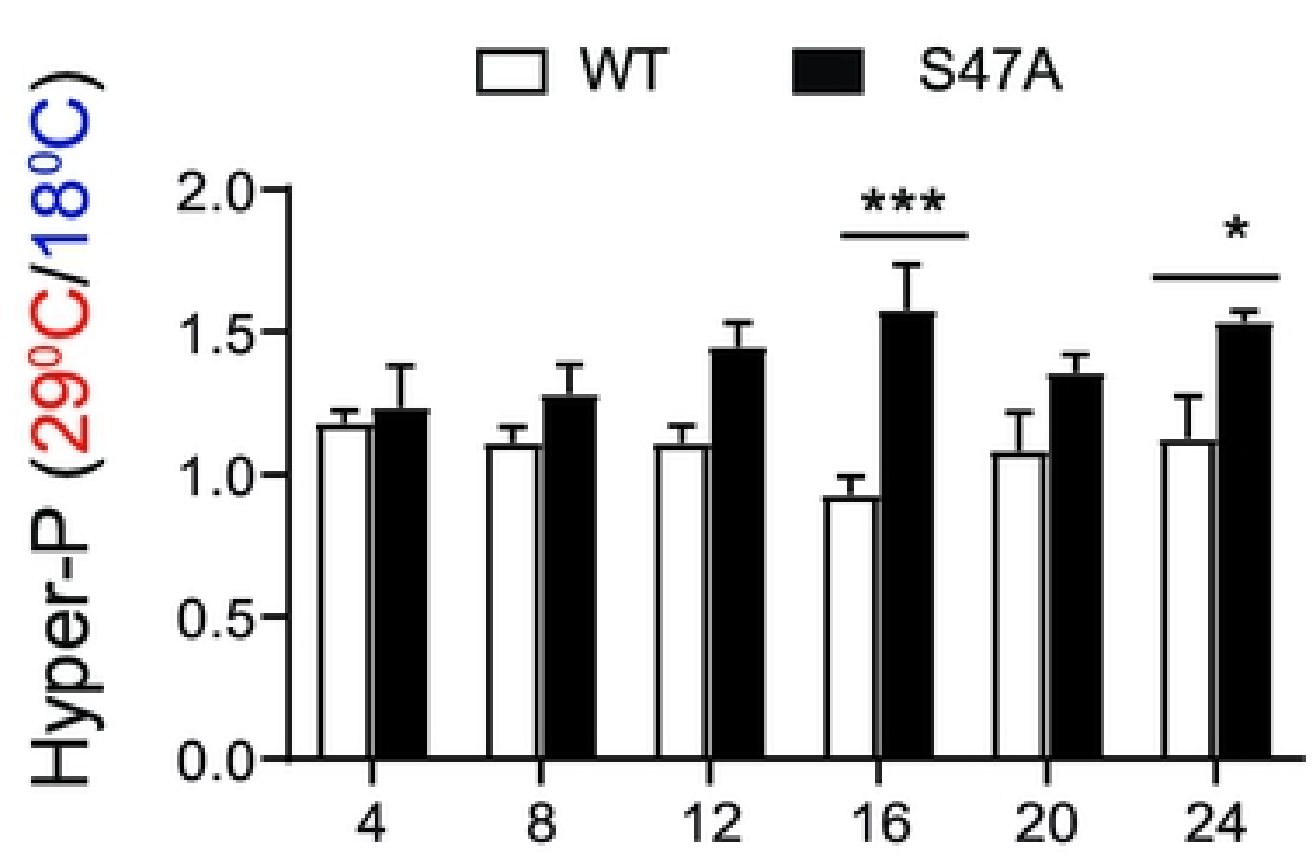

$\operatorname{per}(\mathrm{S} 45 \mathrm{~A})$

\begin{tabular}{|c|c|c|}
\hline & $18^{\circ} \mathrm{C}$ & $29^{\circ} \mathrm{C}$ \\
\hline 4 & $\begin{array}{lllll}8 & 12 & 16 & 20 & 24\end{array}$ & $\begin{array}{llllll}4 & 8 & 12 & 16 & 20 & 24\end{array}$ \\
\hline
\end{tabular}

PER

PER

(longer exposure)

Ponceau S

C

${ }^{\circ} \mathrm{C} \bullet 29^{\circ} \mathrm{C}$

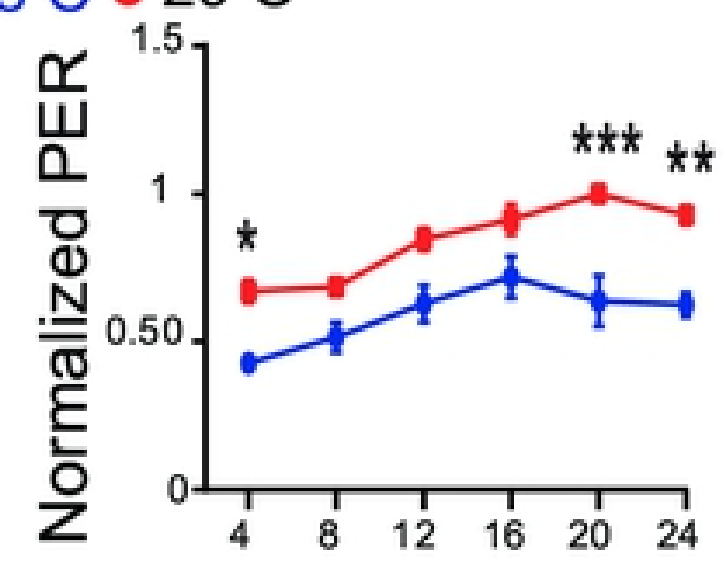

$\mathrm{H}$
Ponceau S
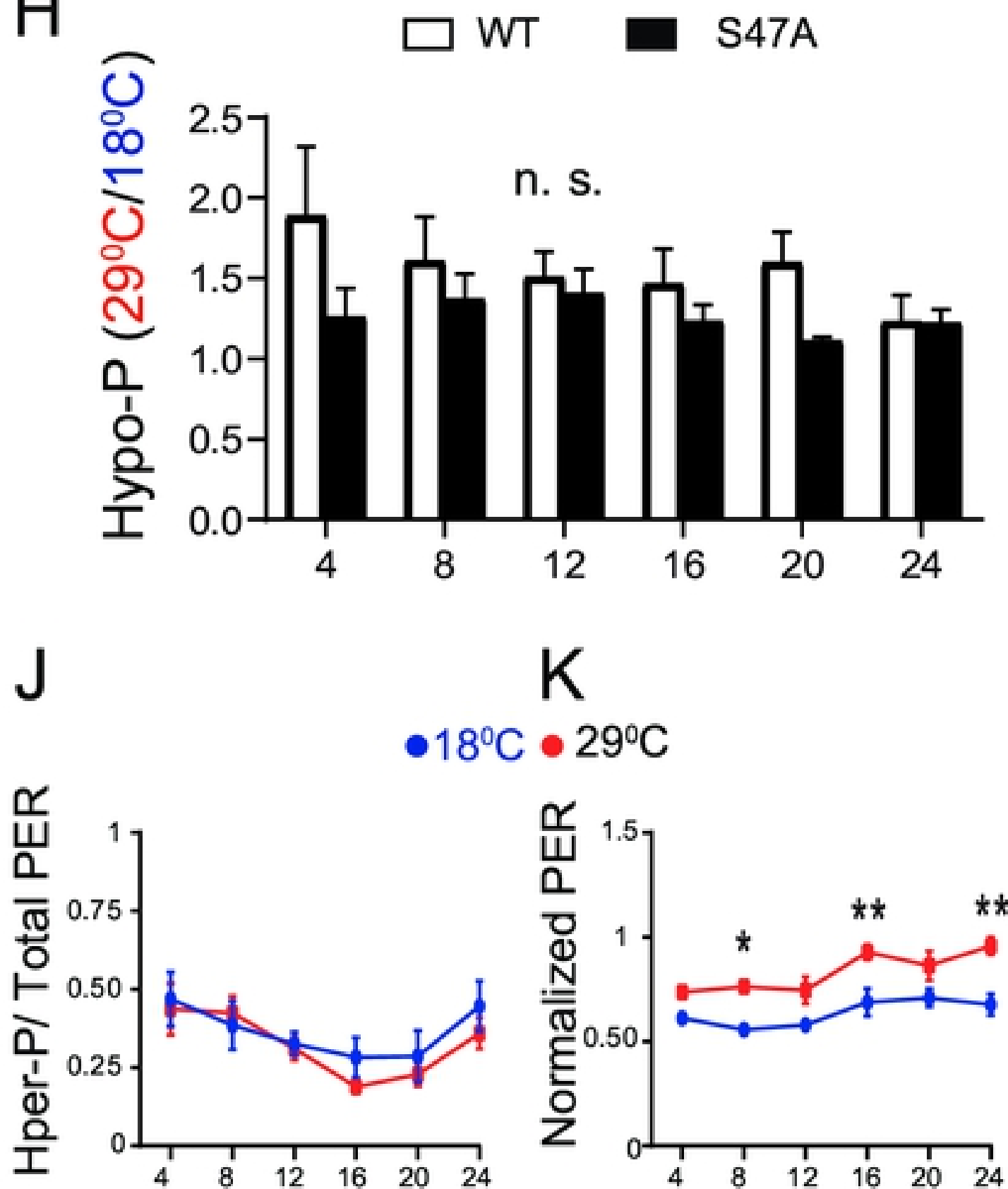

F

$-18^{\circ} \mathrm{C} \bullet 29^{\circ} \mathrm{C}$

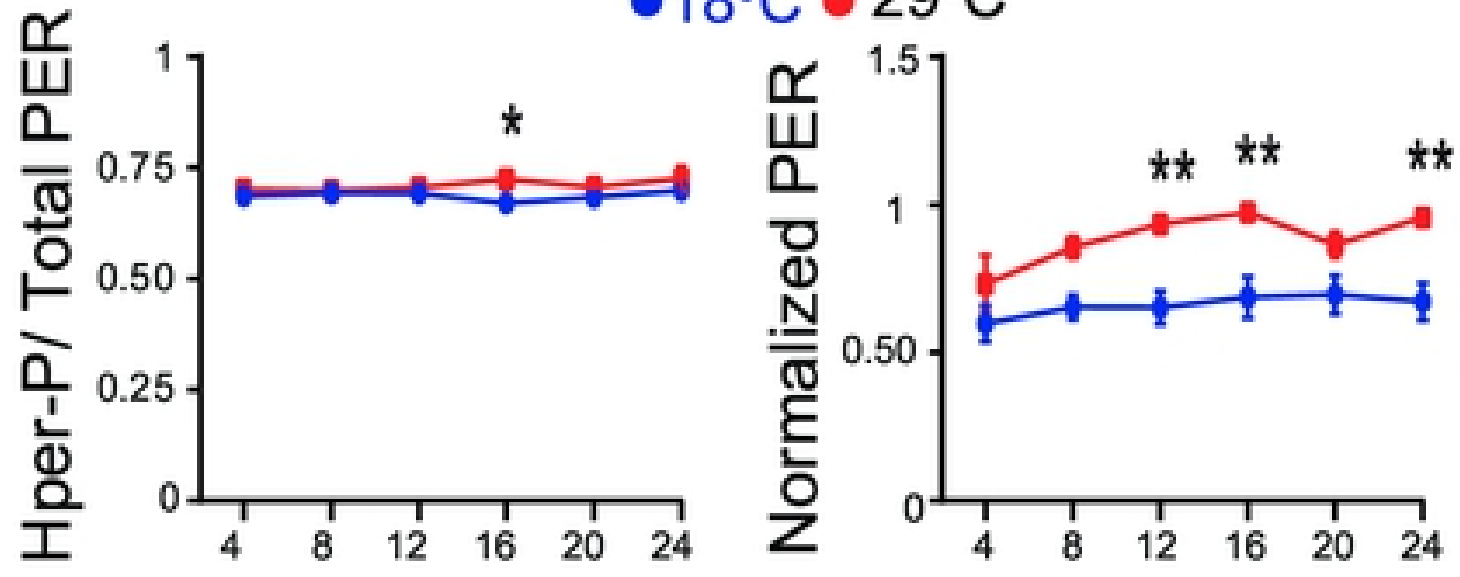

$\mathrm{K}$
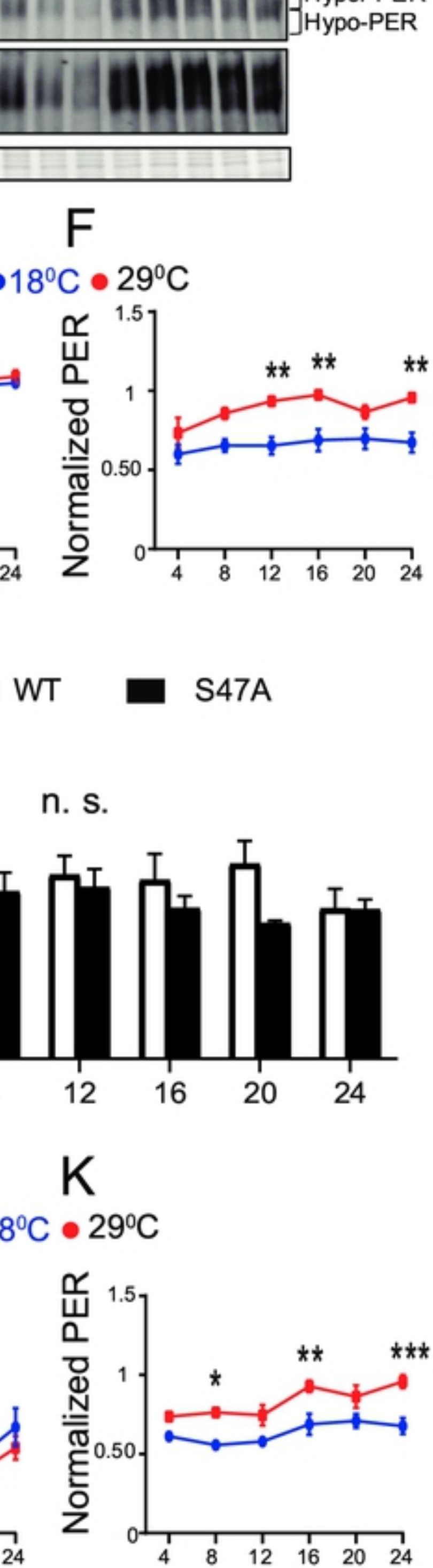

Figure 3 
$A$

Overcompensated

bioRxiv preprint doi: https://doi.org/10.1101/2021.12 23.474078; this version posted December 24, 2021. The copyright holder for this preprint (which was not certified by per review)
perpetuity. It is made author/funder, who has granted bionder uCC-BY 4.0 International license.

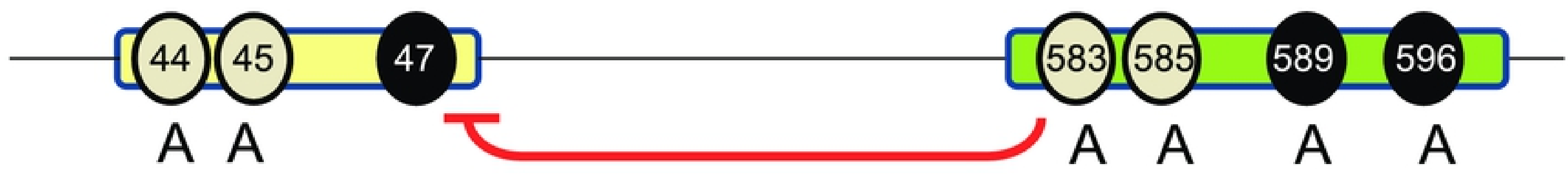

Undercompensated

B

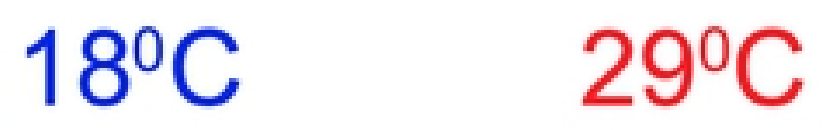

Undercompensated PER degradation

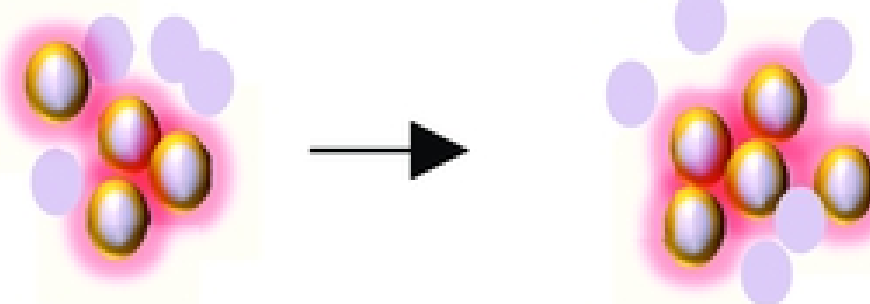

Temperature-compensated circadian clock

\section{PER -WT}

$18^{\circ} \mathrm{C}$

$29^{\circ} \mathrm{C}$

Overcompensated PER degradation

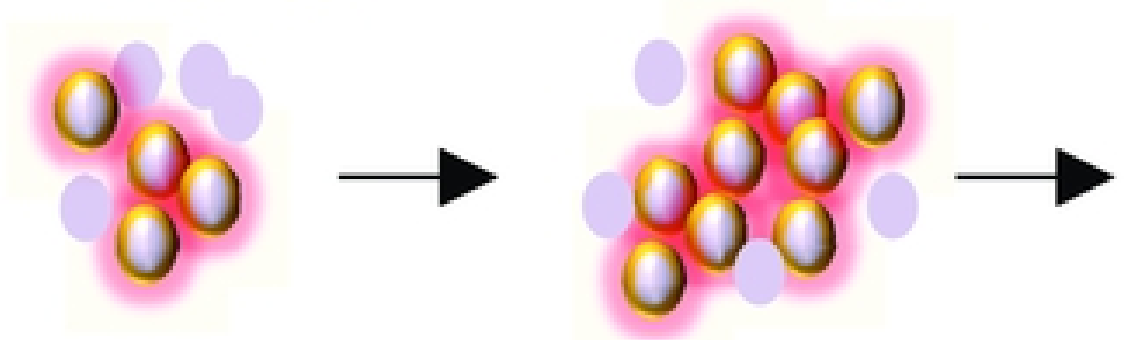

Overcompensated circadian clock

\section{PER-S47A}

Figure 6 
Figure 5

A

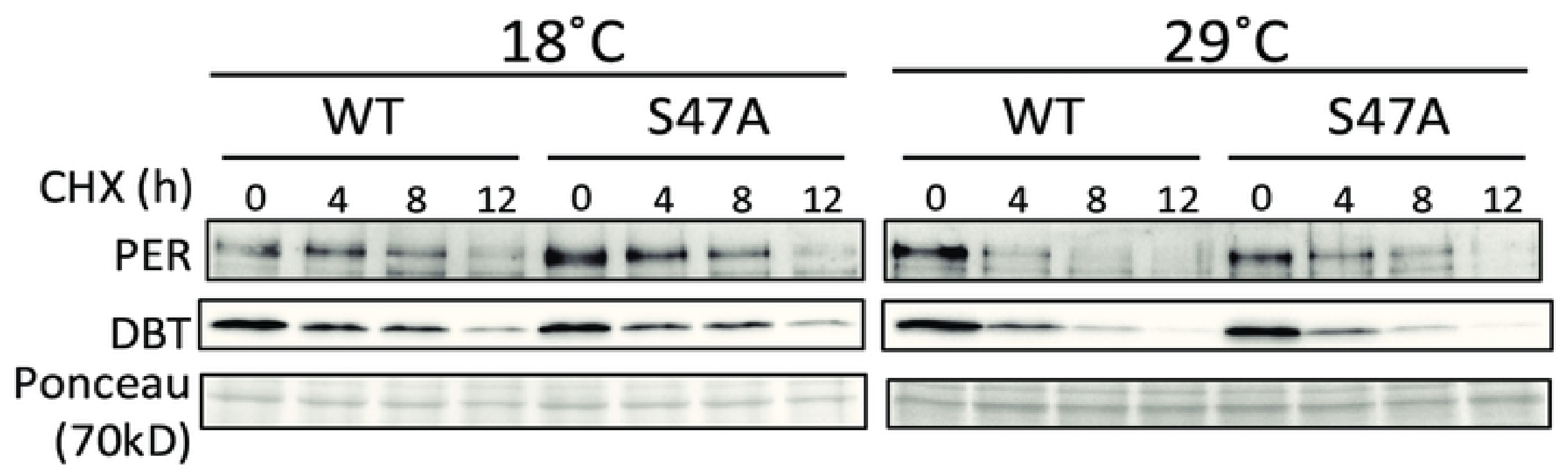

B

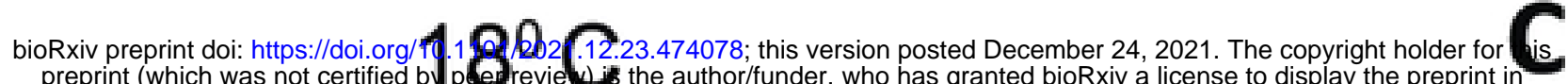
perpetuity. It is made available under aCC-BY 4.0 International license.

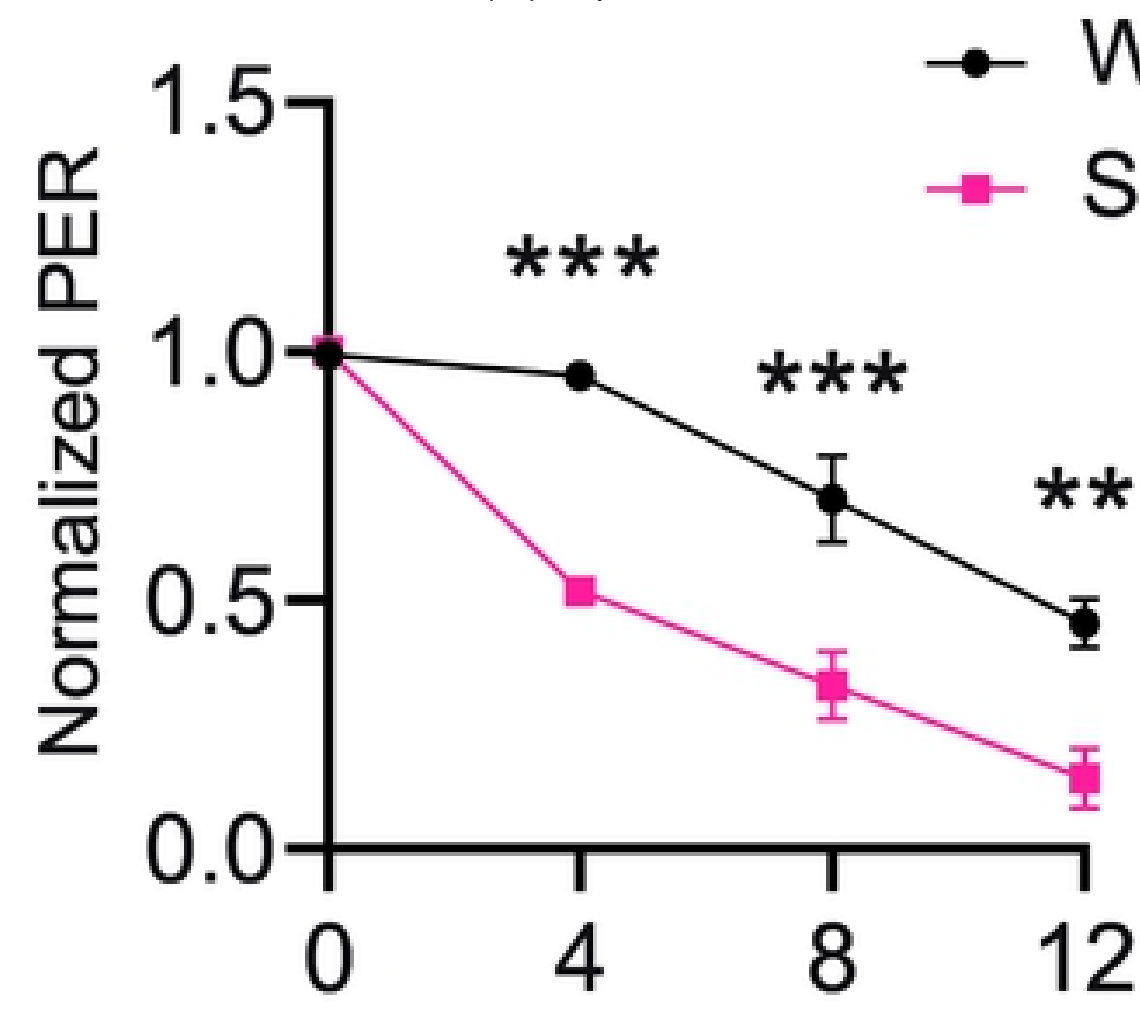

Hours

D

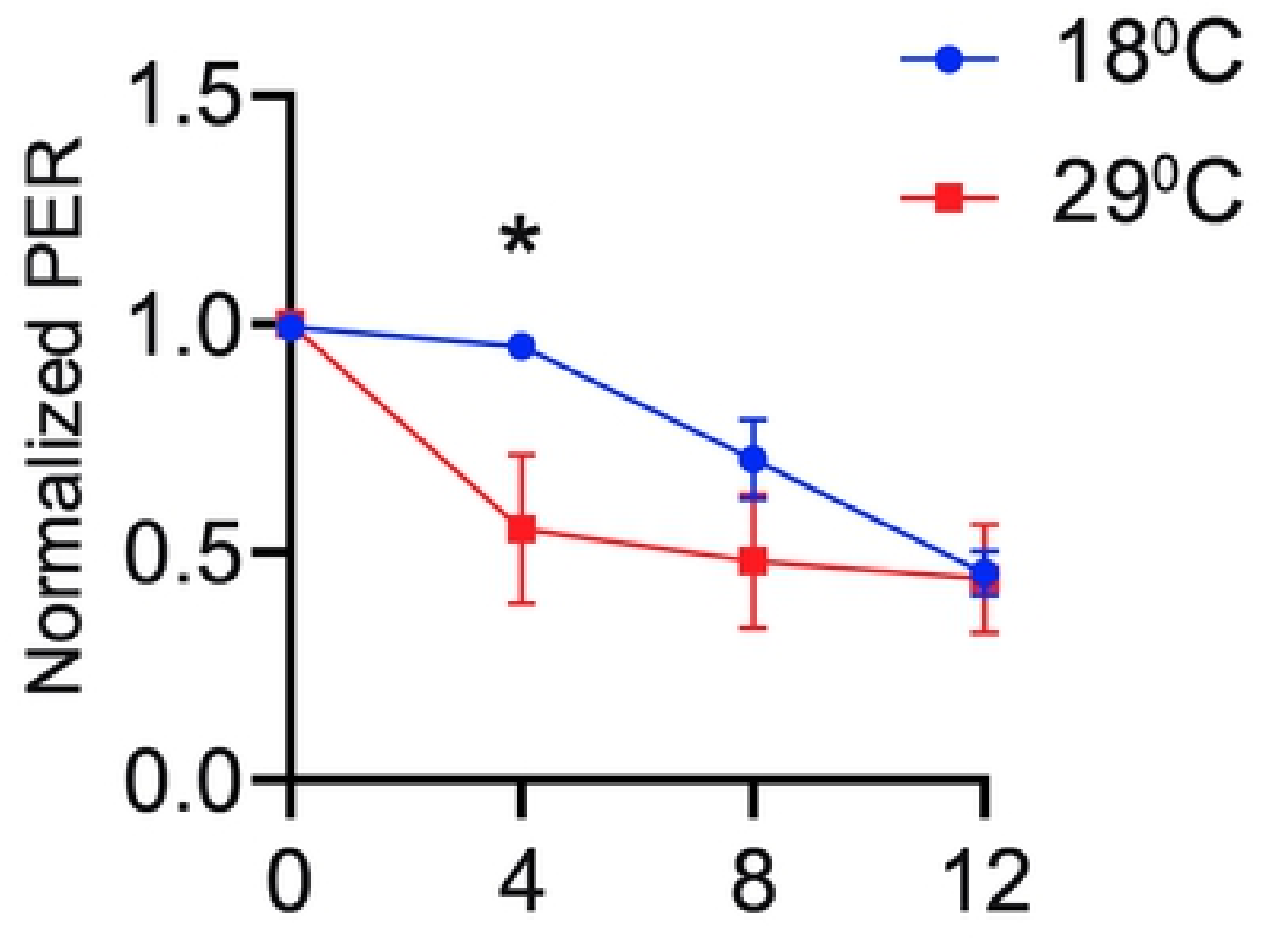

Hours $29^{\circ} \mathrm{C}$

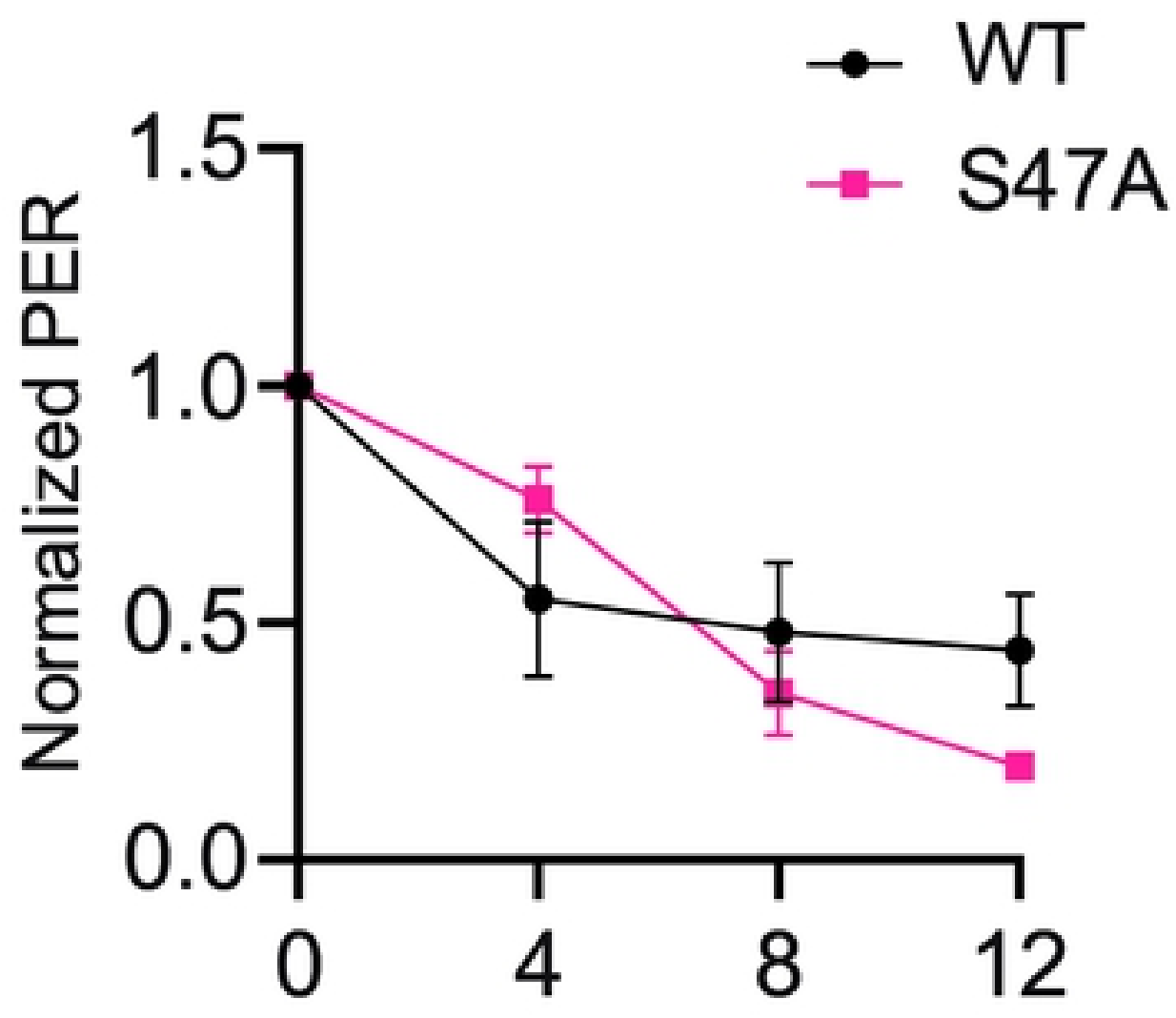

Hours

E S47A

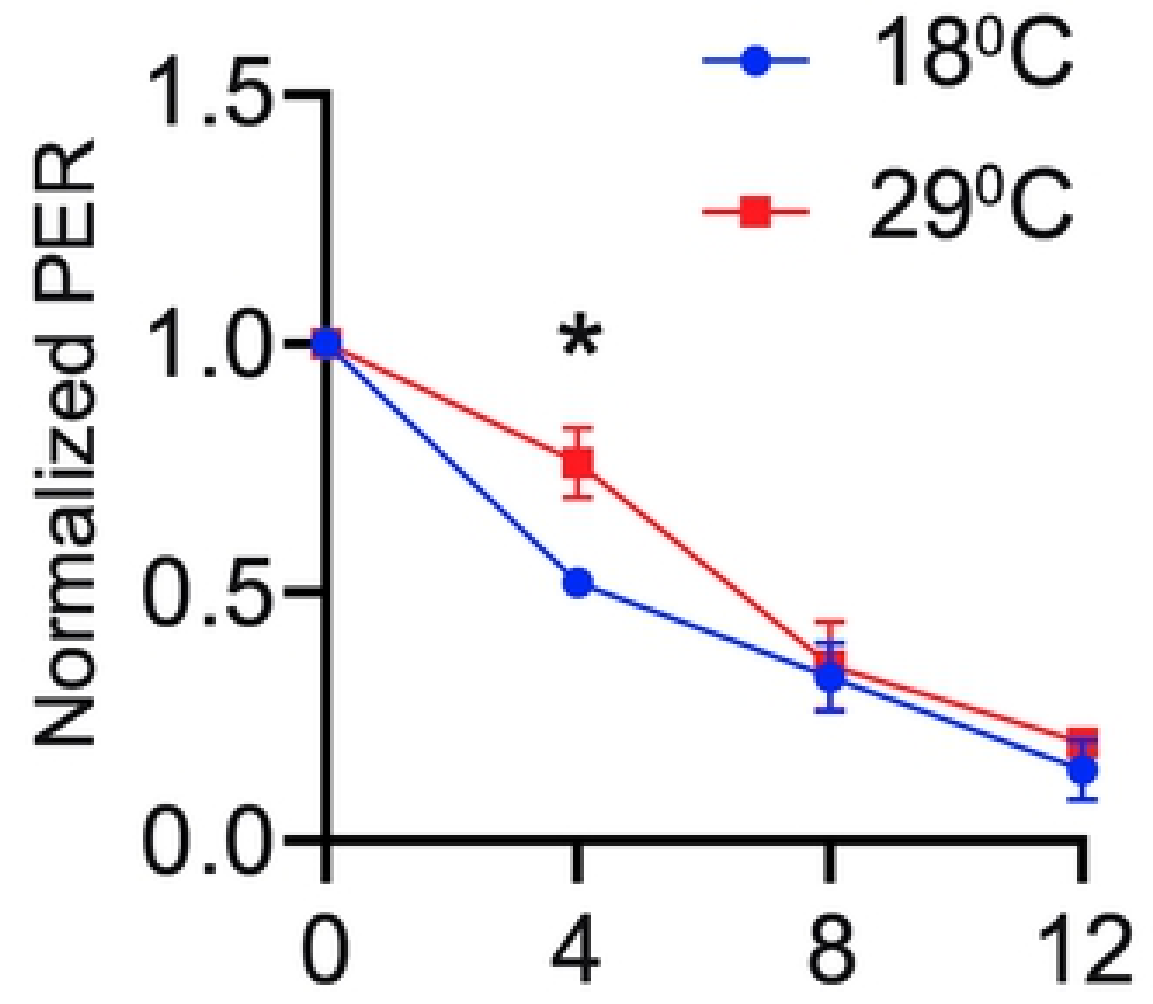

Hours

Figure 5 